\title{
CHARACTERISTICS OF DAMAGED ASPHALT MIXTURES IN TENSION AND COMPRESSION ${ }^{1}$
}

\author{
Robert L. Lytton, Ph.D., P.E. \\ Professor, Fred J. Benson Chair \\ Zachry Department of Civil Engineering \\ Texas A\&M University
}

3136 TAMU, CE/TTI Bldg. 503A, College Station, Texas 77843

Phone: (979) 845-9964, Email: r-lytton@civil.tamu.edu

Yuqing Zhang, Ph.D.

Lecturer

Aston Institute of Materials Research

School of Engineering \& Applied Science

Aston University

MB153A, Aston Triangle, Birmingham, B4 7ET, U.K.

Phone: +44 (0) 121-204-3391, Email: y.zhang10@aston.ac.uk

Fan Gu, Ph.D.

Postdoctoral Researcher

National Center for Asphalt Technology

Auburn University

277 Technology Parkway, Auburn, Alabama 36830

Phone: (334) 844-6251, Email: fzg0014@auburn.edu

Xue Luo, Ph.D.

Assistant Research Scientist

Texas A\&M Transportation Institute

Texas A\&M University System

3135 TAMU, CE/TTI Bldg. 508B, College Station, Texas 77843

Phone: (979) 458-8535, Email: xueluo@tamu.edu

\footnotetext{
${ }^{1}$ This is an Accepted Manuscript of an article published by International Journal of Pavement Engineering. The published article is available at https://doi.org/10.1080/10298436.2017.1347439
} 


\section{Abstract}

This paper addresses the measurement and modeling of the damaged properties of asphalt mixtures including the fracture, healing and viscoplastic deformation of the asphalt mixtures in both tensile and compressive loading as being affected by their composition and conditioning with aging and exposure to temperature and moisture.

An energy-based mechanics is applied to obtain the material fundamental properties such as surface energies, bond energies, anisotropy, yield functions and plastic potential functions that are valid for actual asphalt mixtures, viscoelastic crack growth criteria under both tensile and compressive loading, a simple mechanics-based method of determining the fatigue endurance limit, and the measurement and prediction of healing in restoring the damage done by fracture. Healing is anti-fracture and cracking is the net result of the interplay of these two complimentary mechanisms. Because fracture in asphalt mixtures is not the growth of a single crack but the simultaneous growth of multiple cracks that start out as air voids, this fact leads to the use of the growth of damage density to characterize fracture in an asphalt mixture. It was discovered that the form of Paris' law applies to the growth of damage density of asphalt mixtures in both tensile and compressive loadings. The importance of this fact lies in many developments from this discovery, e.g., compressive monotonic loading of cylindrical test samples permits a direct determination of the Paris’ Law coefficient and exponent.

In all cases measured material properties are presented as they vary with mixture composition and with conditioning such as moisture and aging, both in the lab and in the field. The measurements of these properties are made simply, quickly and accurately by the use of mechanics so that an entire characterization of the properties of an asphalt mixture in tension and compression can be completed in the space of one day. The net effect is to reduce the efforts expended in the lab and the systematic error due to the assumptions made by the existing models and simultaneously to increase the efficiency and cost-effectiveness of materials testing and raise the reliability of the design of mixtures, pavement structures and specifications and the prediction of the life cycles in as-built pavements. 


\section{Introduction}

This is the second paper in a sequence that discusses and illustrates how the properties of asphalt mixtures are measured. The properties in the undamaged state were presented in the first paper, entitled "Characteristics of undamaged asphalt mixtures in tension and compression”. This paper addresses the measurement and modeling of the damaged properties of asphalt mixtures. The two papers together constitute a roadmap with references to the many previously published works which explain and illustrate the concept of energybased mechanics.

The content of this paper is more specific than that is implied with the word "damage". In this paper, “damage” means the fracture, healing, and viscoplastic deformation of asphalt mixtures in both tensile and compressive loading, referring to the actual physical changes that occur in asphalt mixtures caused by the application of externally applied energy. This is in contrast to the more generically applied term which refers to an empirically-derived explanation of the departure of the observed response of a material from the response of an undamaged material to the application of externally applied energy. The distinction is important. The purpose of characterizing the damaged properties of an asphalt mixture is to use those characteristics in the design of mixtures and pavement structures, as well as in construction specifications and predictions of the expected life of the pavements built with these mixtures. The designs, specifications and predictions carry with them a required reliability which must be adequate for the service for which they are intended. Reliability requires the use of both the mean and the variance of the measurements that are made. Variance is made up of two possible sources: systematic and random error. Random error can be reduced by repeating the measurement. Systematic error comes from assuming that the explanation of a measurement is one thing when the actual explanation is another. The only way to reduce systematic error is to correct the assumed explanation.

However, much testing of asphalt mixtures' damage properties is based on an assumed phenomenological relationship between a calculated response and an observed result. The relationship relies on the assumption that to "simulate and correlate" will reveal all of the needed materials information. Such correlations usually have very large variances. When these large variances are factored into the reliability-based design of mixtures, pavement structures or specifications, the results are generally unacceptably impractical.

Thus there is a very practical aim of being accurate and precise in defining the actual "damage" in physical terms and that is to reduce systematic error, to reduce the size of the 
variance of the test results and, as a consequence, to raise the level of reliability while remaining within practical limits. The terms "accuracy" and "precision" are thus meant in the mechanics sense: the ability to measure, with small variances, real material properties that describe actual physical changes in an asphalt mixture such as fracture, healing, and viscoplastic deformations. In sum, the purpose of using mechanics is to reduce systematic error and to make the measurement of the relevant material properties simple, rapid, efficient, accurate and precise. Instead of using a "simulate and correlate” approach, this approach emphasizes "cause and effect” which is the essence of mechanics.

In this paper, the reader will firstly find illustration of measured material properties such as surface energies and bond energies. Then the constitutive and characterizing models for asphalt mixtures' damage are presented including anisotropy, yield functions and plastic potential functions that are valid for actual asphalt mixtures, viscoelastic crack growth criteria under both tensile and compressive loading, a simple mechanics-based method of determining the endurance limit, and the measurement and prediction of healing in restoring the damage done by fracture. The last section shows the measured material properties that are presented as they vary with mixture composition and with conditioning such as moisture and aging.

\section{Bond Energy in Asphalt Mixtures}

\subsection{Cohesive Fracture and Adhesive Fracture}

The bond energy of an asphalt mixture is an important property that dominates the crack initiation and propagation processes. It is known that the bond energy can be a cohesive bond energy when the crack occurs within a single material phase or an adhesive bond energy when the crack propagates along the interface of two material phases, which are expressed as below (Cheng et al., 2002):

$$
\left\{\begin{array}{l}
\Delta G_{i}^{c}=2 \gamma_{i}=2\left(\gamma_{i}^{L W}+\gamma_{i}^{A B}\right)=2\left(\gamma_{i}^{L W}+2 \sqrt{\gamma_{i}^{+} \gamma_{i}^{-}}\right) \\
\Delta G_{i j}^{a}=2\left(\gamma_{i}+\gamma_{j}-\gamma_{i j}\right)=2 \sqrt{\gamma_{i}^{L W} \gamma_{j}^{L W}}+2 \sqrt{\gamma_{i}^{+} \gamma_{j}^{-}}+2 \sqrt{\gamma_{i}^{-} \gamma_{j}^{+}}
\end{array}\right.
$$

where $\Delta G_{i}^{c}$ and $\Delta G_{i j}^{a}$ are cohesive and adhesive bond energy, respectively. The subscript i and $\mathrm{j}$ stand for material i (e.g., asphalt mastic) and material $\mathrm{j}$ (e.g., aggregate). $\gamma_{i}$ is the surface free energy of the material $i, \gamma_{i}^{L W}$ is the Lifshitz-van der Waals (LW) surface energy component of the material $\mathrm{i}, \gamma_{i}^{A B}$ is the acid-base $(\mathrm{AB})$ surface energy component of the material i, $\gamma_{i}^{+}$and $\gamma_{i}^{-}$are the Lewis acid and base components of the material i. $\gamma_{i j}$ is the interfacial surface free energy between material $\mathrm{i}$ and material $\mathrm{j}$. 
According to the study in the literature (Lytton et al. 2005), fracture in asphalt mixtures can occur within the asphalt (cohesive fracture) or at the aggregate-asphalt interface (adhesive fracture), which depends on the asphalt binder property, the asphalt film thickness, the temperature and the loading rate. Cohesive fracture is preferred in thick asphalt films whereas adhesive fracture is preferential in thinner asphalt films (Marek and Herrin 1968). Since the film thicknesses varies in asphalt mixtures, adhesive and cohesive fracture can occur simultaneously in one asphalt mixture. Therefore, the bond energy for the asphalt mixture is neither a pure cohesive bond energy of asphalt nor a pure adhesive bond energy between asphalt and aggregates, but an integrated representative bond energy of the cohesive and adhesive fracture. A decomposition of the bond energy of the asphalt mixture is formulated as (Zhang et al. 2014c),

$$
\Delta G=\alpha \cdot \Delta G^{c}+(1-\alpha) \Delta G^{a}
$$

where $\Delta G^{c}$ is the cohesive bond energy within the asphalt, $\Delta G^{a}$ is the adhesive bond energy at the asphalt-aggregate interface, $\alpha$ varies from 0 to 1 , interpreted as the percentage of cohesive fracture in an asphalt mixture. The paper (Zhang et al. 2014c) presents the method for determining $\alpha$.

\subsection{Measurement of Surface Energy}

Surface energy of aggregate is measured using the Universal Sorption Device (USD), while surface energy of asphalt binder is measured using the Wilhelmy plate. The USD indirectly determines the aggregate surface energy by using gas adsorption characteristics of three solvents. The Wilhelmy plate determines the surface energy of asphalt binder by using the contact angle between a thin plate coated with binder immersed and withdrawn from a liquid with known surface energy components. The detailed procedures of the USD test and Wilhelmy plate test can be found in Lytton et al. (2005). Once all of the surface energy components for both asphalt binder and aggregate are obtained, the bond energy of an asphalt mixture can be calculated using Equation 1. The bond energy of an asphalt mixture is not only dependent on the surface energies of the asphalt and aggregate, but also is influenced by the presence of water. With the presence of the water, the bond energy of an asphalt mixture is calculated by the following equation (Tong 2013):

$$
\begin{aligned}
\Delta G_{i k j}= & \sqrt{\gamma_{i}^{L W} \gamma_{k}^{L W}}+\sqrt{\gamma_{j}^{L W} \gamma_{k}^{L W}}-\sqrt{\gamma_{i}^{L W} \gamma_{j}^{L W}}-\gamma_{k}^{L W}+2 \sqrt{\gamma_{k}^{+}}\left(\sqrt{\gamma_{i}^{-}}+\sqrt{\gamma_{j}^{-}}-\sqrt{\gamma_{k}^{-}}\right)+ \\
& 2 \sqrt{\gamma_{k}^{-}}\left(\sqrt{\gamma_{i}^{+}}+\sqrt{\gamma_{j}^{+}}-\sqrt{\gamma_{k}^{+}}\right)-2 \sqrt{\gamma_{i}^{+} \gamma_{j}^{-}}-2 \sqrt{\gamma_{i}^{-} \gamma_{j}^{+}}
\end{aligned}
$$


where subscribes i, j, and k represent asphalt binder, aggregate, and water, respectively.

Figures 1a and 1b compare the adhesive bond energy for different asphalt mixtures with and without the presence of water. The adhesive bond energy of asphalt mixtures varies significantly for the different types of aggregate and binder. Four types of warm mix asphalt (WMA) additives (i.e., Advera, Sasobit, Evotherm and Rediset) are added to the virgin binder, which effectively reduce the adhesive bond energy of asphalt mixtures. When the interface between an asphalt binder and aggregate is in contact with water, the bond energy changes from a positive value to a negative value. This indicates that the water plays a role in debonding the interface between asphalt binder and aggregate.

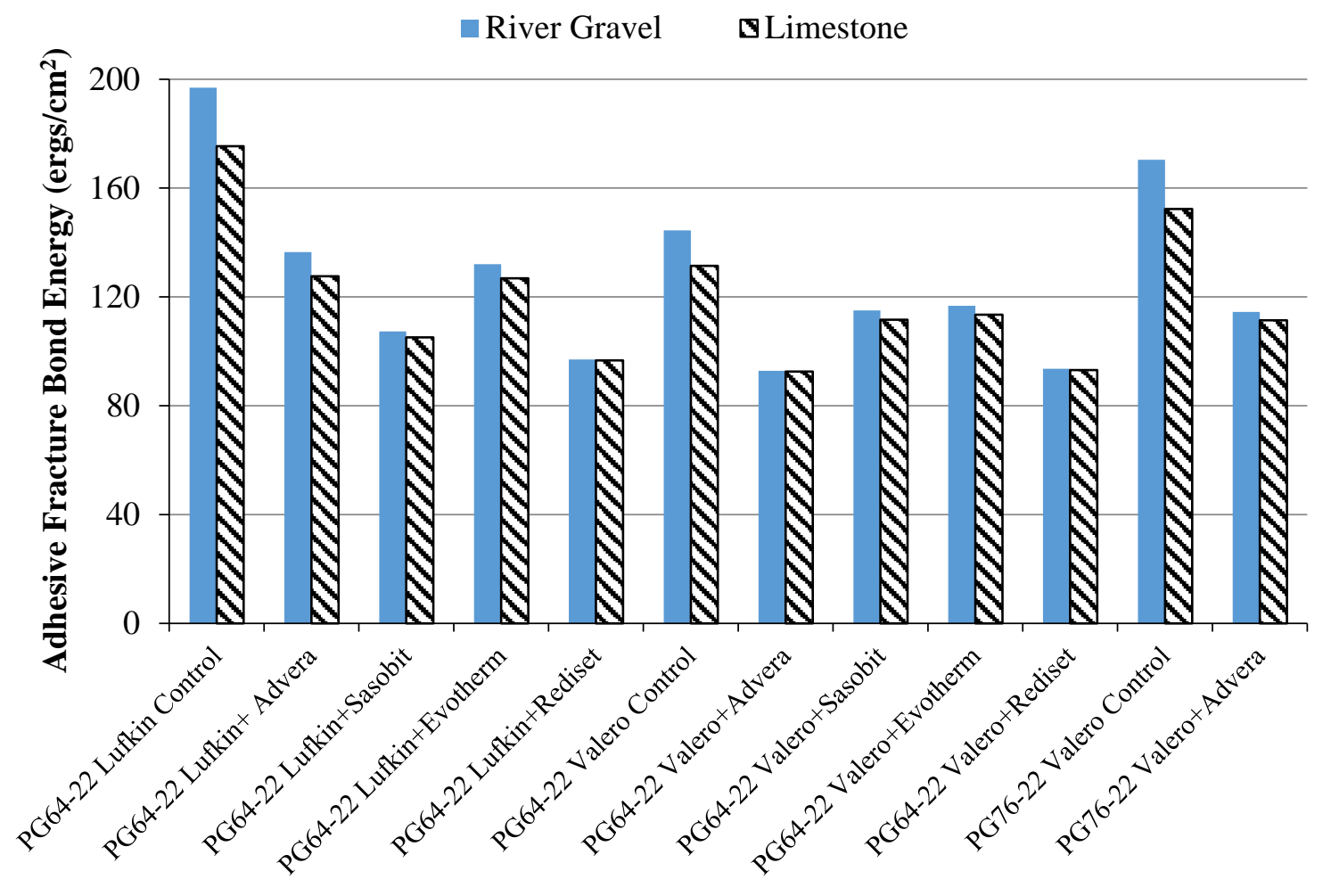

a. Adhesive Bond Energy of Asphalt Mixtures without Water 


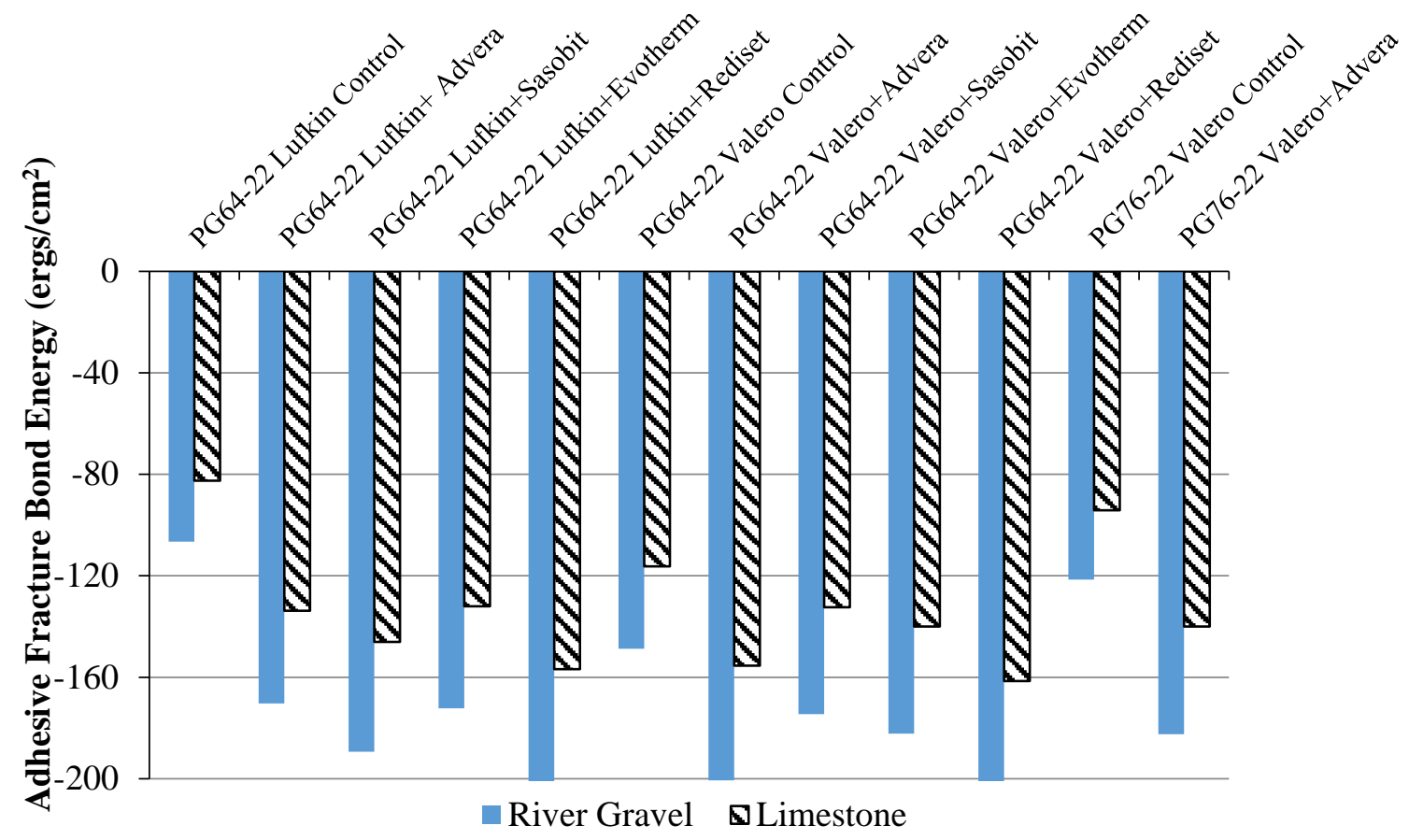

b. Adhesive Bond Energy of Asphalt Mixtures with Water

Figure 1. Comparison of Adhesive Bond Energy for Different Asphalt Mixtures with and without Water

Figure 2 shows the effect of water on the cohesive bond energy of asphalt mixtures. The presence of water significantly decreases the cohesive bond energy of asphalt mixtures, which will further reduce the fatigue cracking resistance of asphalt mixtures. 


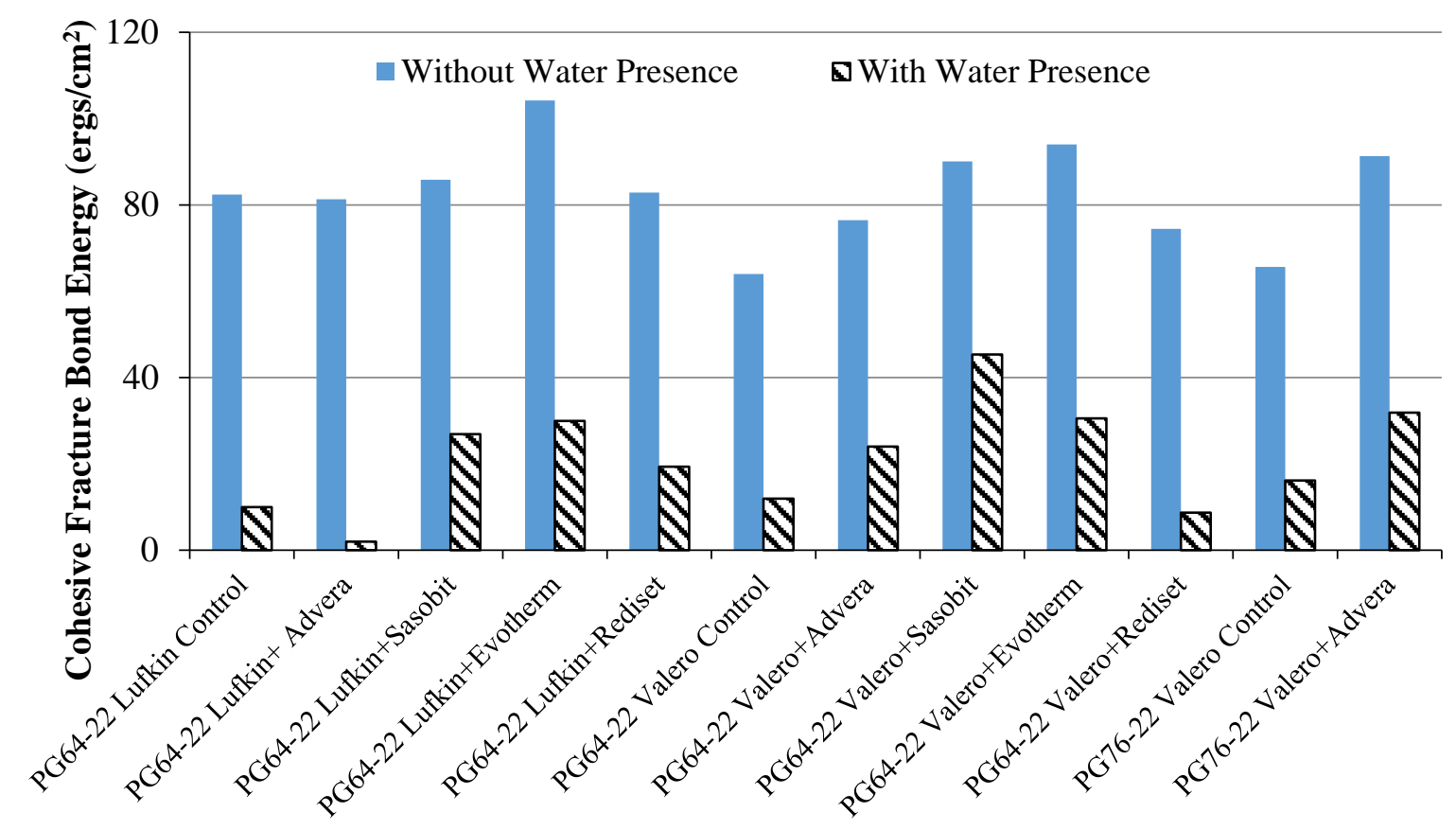

Figure 2. Comparison of Cohesive Bond Energy for Different Asphalt Mixtures with and without Water

\section{Viscoelastic-plastic Fracture Constitutive Models for Asphalt Mixtures}

\subsection{Cracking Damage Characterization}

Once a material is damaged, the load applied to the material is transferred via the remaining undamaged (effective or intact) material. It is argued that an effective stress acting on the intact material drives the viscoelastic and viscoplastic deformation (Lemaitre and Desmorat 2005; Kachanov, 1986). Thus the constitutive equations are valid only in the effective (undamaged) configuration and the stresses $\left(\sigma_{i j}\right)$ used in these models should be the effective stresses. However, the stresses measured on the damaged material are apparent stresses $\left(\sigma^{A} i j\right)$ that are affected by cracking damage, and the apparent modulus also degrades with crack evolution. Based on continuum damage mechanics (CDM) (Lemaitre and Desmorat 2005; Chaboche 1987), a damage density is defined as below to capture the overall fracture properties and relate the apparent responses to the effective responses and material properties.

$$
\xi=A^{L} / A^{T}
$$

where, $\xi$ = damage density, $A^{L}=$ lost area due to damages such as voids, flaws and cracks and $A^{T}=$ total cross-sectional area of the material. According to the CDM theories, two equilibrium principles (i.e., force and energy) and isotropic damage are hypothesized between 
the undamaged (effective) configuration and the damaged (apparent) configuration, leading to:

$$
\left\{\begin{array}{l}
\sigma_{i j}^{A}=\sigma_{i j}(1-\xi) \\
\varepsilon_{i j}=\varepsilon_{i j}^{A}(1-\xi)
\end{array}\right.
$$

where $\sigma_{i j}$ and $\varepsilon_{i j}=$ effective stress and strain defined in the effective configuration; $\sigma^{A} i j$ and $\varepsilon^{A}{ }_{i j}$ $=$ apparent stress and strain defined in the apparent configuration and measured in the laboratory.

Similarly, in a three dimensional condition considering anisotropy, the anisotropic damage density can be defined as:

$$
\xi_{i j}=\left[\begin{array}{ccc}
\xi_{1} & 0 & 0 \\
0 & \xi_{2} & 0 \\
0 & 0 & \xi_{2}
\end{array}\right]=\left[\begin{array}{ccc}
A_{1}^{L} / A_{1}^{T} & 0 & 0 \\
0 & A_{2}^{L} / A_{2}^{T} & 0 \\
0 & 0 & A_{2}^{L} / A_{2}^{T}
\end{array}\right]
$$

where $\xi_{1}$ and $\xi_{2}$ are axial and radial damage density, respectively; $A_{1}^{L}$ and $A_{2}^{L}$ are the projected lost area in the axial and radial directions, respectively; $A_{1}^{T}$ and $A_{2}^{T}$ are the total area in the axial and radial directions, respectively. To formulate the true stress tensor based on the anisotropic damage densities, a symmetric fourth-order damage density tensor is employed as follows (Voyiadjis and Park, 1997):

$$
M_{i m n j}=\frac{1}{2}\left[\delta_{i m}\left(\delta_{n j}-\xi_{n j}\right)^{-1}+\left(\delta_{i m}-\xi_{i m}\right)^{-1} \delta_{n j}\right]
$$

where $M_{i m n j}$ is the fourth-order damage density tensor; $\delta_{i j}$ is the Kronecker delta tensor.

Then the true stress tensor ( $\sigma_{i j}^{T}$ ) becomes (Abu Al-Rub and Voyiadjis, 2003):

$$
\sigma_{i j}=M_{i m n j} \sigma_{m n}^{A}=\frac{1}{2}\left[\sigma_{i n}^{A}\left(\delta_{n j}-\xi_{n j}\right)^{-1}+\left(\delta_{i m}-\xi_{i m}\right)^{-1} \sigma_{m j}^{A}\right]
$$

where $\sigma_{m n}^{A}$ is the apparent stress tensor. The true stress tensor $\left(\sigma_{i j}\right)$ is capable of accounting for the crack-induced anisotropy of the asphalt mixtures by applying the true stress tensor and the anisotropic damage densities in the constitutive modeling of the material.

\subsection{Viscoplastic Damage Models}

The viscoplastic strain is associated with irrecoverable permanent deformation of the material and its evolution can be defined by a Perzyna-type viscoplasticity theory as (Perzyna 1971): 


$$
\dot{\varepsilon}_{i j}^{v p}=\Gamma\langle\Phi(f)\rangle^{N} \frac{\partial g}{\partial \sigma_{i j}}
$$

where $\dot{\varepsilon}_{i j}^{v p}=$ rate of the viscoplastic strain with respect to time; $\Gamma=$ a viscosity related parameter. $N=$ a viscoplastic rate dependent exponent. Both $\Gamma$ and $N$ are experimentally determined and $N>1$ for the asphalt mixtures. $f=$ a viscoplastic yield surface function. $g=\mathrm{a}$ viscoplastic plastic potential function. The non-associated flow rule applies that $g \neq f$, which is appropriate for shearing dilated geo-materials such as asphalt mixtures. $\Phi(f)=f / P a$, The McCauley brackets imply that:

$$
\langle\Phi(f)\rangle= \begin{cases}0, & \Phi(f) \leq 0 \\ f / P a, & \Phi(f)>0\end{cases}
$$

The Perzyna-type model in Equation 9 has been used to describe asphalt mixture viscoplastic evolution for decades (Abdulshafi and Majidzadeh 1984; Tan et al. 1994; Seibi et al. 2001; Tashman et al. 2005; Masad et al. 2007; Abu Al-Rub et al. 2012). However, most used a yield surface like Mohr-Coulomb, Drucker-Prager (D-P) or extended D-P models which have some significant limitations such as non-smooth and/or non-convex surfaces when the internal frictional angle is greater than $22^{\circ}$. To remove these limitations, the authors developed a generalized Drucker-Prager (GD-P) yield surface model as (Zhang et al. 2014a; Zhang 2012):

$$
f=\sqrt{J_{2}} \rho\left(\theta^{\prime}\right)-\alpha I_{1}-\kappa a_{k} a_{\varepsilon}
$$

where $J_{2}=1 / 2 s_{i j} S_{i j}=$ the second invariant of the deviatoric stress tensor $\left(s_{i j}\right) ; I_{1}=\sigma_{k k} . \theta^{\prime}=$ the Lode angle that is defined as:

$$
\theta^{\prime}=\frac{1}{3} \arccos \left[\frac{3 \sqrt{3}}{2} \frac{J_{3}}{\left(J_{2}\right)^{3 / 2}}\right], \quad \theta^{\prime} \in\left[0, \frac{\pi}{3}\right]
$$

where $J_{3}=\operatorname{det}\left(s_{i j}\right)=$ the third invariant of the deviatoric stress tensor. $\theta^{\prime}$ is zero in compression and $\pi / 3$ in extension. $\rho\left(\theta^{\prime}\right)$ is a function defining the yield surface shape on the octahedral plane and determines the convexity of the yield surface, which is expressed as:

$$
\rho\left(\theta^{\prime}\right)=\mu \cos \left[\frac{1}{3} \arccos \left(\gamma \cos 3 \theta^{\prime}\right)\right]
$$


where $\mu$ and $\gamma$ are size and shape parameters, respectively, for the yield surface on the octahedral plane to ensure that the yield surface is smooth and convex, which are computed by:

$$
\mu=\frac{2 \sqrt{1-d+d^{2}}}{\sqrt{3} d}, \gamma=-\frac{3 \sqrt{3}}{2} \frac{(1-d) d}{\left(1-d+d^{2}\right)^{1.5}}, \quad d=\frac{3-\sin \phi}{3+\sin \phi}
$$

where $d=$ an extension ratio that is the ratio of the yield strength in extension to that in compression. $\phi=$ internal friction angle of the asphalt mixture, which also directly determines the slope of the yield surface on the meridian plane by:

$$
\alpha=\frac{2 \sin \phi}{\sqrt{3}(3-\sin \phi)}
$$

Figure 3 plots three dimensional (3-D) GD-P yield surfaces with different internal friction angles. One can find that the GD-P model always provides a smooth and convex yield surface when the internal friction angle varies from $0^{\circ}$ to $90^{\circ}$. The yield strength in extension is less than that in compression, which is quantified by the extension ratio, i.e., $d$. It can be proved that the GD-P yield surface coincides with the apices of the Mohr-Coulomb yield surface on the octahedral plane.

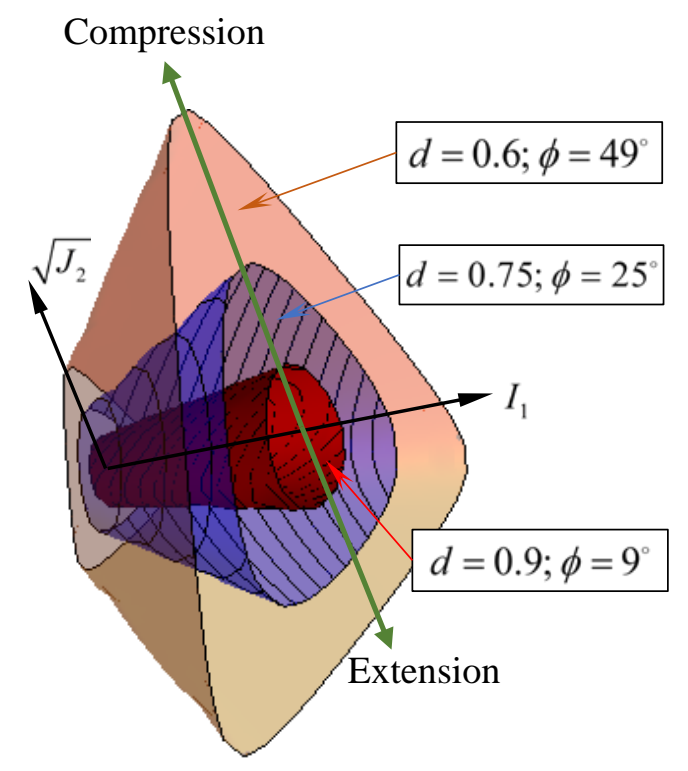

\section{Figure 3. Three Dimensional Plot of Generalized Drucker-Prager (GD-P) Yield Surfaces}

The term $\kappa a_{k} a_{\varepsilon}$ in the GD-P model represents the temperature and strain-rate dependent cohesion and strain hardening for the asphalt mixture. $\kappa=$ a strain hardening function; $a_{k}$ and $a_{\varepsilon}$ are temperature and strain-rate effect factors, and they are defined as: 


$$
\begin{aligned}
& \kappa=\kappa_{0}+\kappa_{1}\left[1-\exp \left(-\kappa_{2} \varepsilon_{e}^{v p}\right)\right] \\
& a_{k}(T)=\exp \left[\frac{\Delta E_{k}}{R}\left(\frac{1}{T}-\frac{1}{T_{r}}\right)\right] \\
& a_{\varepsilon}(\dot{\varepsilon})=\left(\dot{\varepsilon} / \dot{\varepsilon}_{r}\right)^{\kappa_{3}}
\end{aligned}
$$

where $\kappa_{0}$ is a parameter determined by material cohesion $(C)$ and internal friction angle $(\phi)$ :

$$
\kappa_{0}=\frac{6 C \cos \phi}{\sqrt{3}(3-\sin \phi)}
$$

$\kappa_{1}$ and $\kappa_{2}$ are the material parameters identified at the reference temperature $\left(T_{r}\right)$ and a reference strain-rate $\left(\dot{\varepsilon}_{r}\right)$. They determine the size and shape of the strain hardening yield surface, respectively. $\Delta E_{k}=$ the activation energy for the temperature effect on cohesion, $\dot{\varepsilon}=$ a strain rate of interest, and $\kappa_{3}=$ a material property accounting for the effect of strain-rate on cohesion. $\varepsilon e^{v p}=$ an effective viscoplastic strain under multi-axial stress loadings, that is derived as (Zhang 2012):

$$
\dot{\varepsilon}_{e}^{v p}=A_{e} \sqrt{\dot{\varepsilon}_{i j}^{v p} \dot{\varepsilon}_{i j}^{v p}}=\left[\left(\frac{1-\sqrt{3} \beta}{1-\sqrt{3} \alpha}\right)^{2}+2\left(\frac{0.5+\sqrt{3} \beta}{1-\sqrt{3} \alpha}\right)^{2}\right]^{-\frac{1}{2}} \sqrt{\dot{\varepsilon}_{i j}^{v p} \dot{\varepsilon}_{i j}^{v p}}
$$

where $\beta=$ the slope of the viscoplastic potential surface. Integrating Equation 20 over time gives the effective viscoplastic strain. The potential function $(g)$ determines the direction of the viscoplastic strain increment. It is assumed that the viscoplastic potential surface has the same linear form as the yield surface but with a smaller slope which affects the volumetric dilation of the material:

$$
g=\sqrt{J_{2}} \rho\left(\theta^{\prime}\right)-\beta I_{1}
$$

A number of studies have indicated that $\beta<\alpha$ is valid for geo-materials such as soils, sands, and asphalt mixtures (Tashman et al. 2005; Oda and Nakayama 1989). $\beta$ is determined to be a function of anisotropy:

$$
\beta=0.5889 \Delta^{\prime}-0.0122
$$

where $\Delta^{\prime}=$ modified vector magnitude for microstructural anisotropy of geo-materials that has a value from 0.2 to 0.5 and can be experimentally determined in a matter of minutes using a digital scan of the lateral surface of a core or a lab sample. (Zhang et al. 2011). 
Figure 4 shows the slope of the GD-P yield surface $(\alpha)$ that is independent of binder type, air void content and aging period. In the figure, VHL stands for the asphalt mixtures using Valero binder (PG64-16) and Hanson Limestone. NHL are these mixtures made of NuStar binder (PG67-22) and Hanson Limestone. This is reasonable since $\alpha$ is solely determined by the internal friction angle of asphalt mixtures, which relies on the aggregate contacts and interlocks of the aggregate gradation that remain the same in this study. Figure 4 also indicates that the intercept of the GD-P yield surface $\left(\kappa_{0}\right)$ increase as the aging period increases or the air void content decreases. Actually, $\kappa_{0}$ quantifies the cohesive properties of the material. A stiffer asphalt mixture (e.g., due to stiffer binder, low air voids, longer aging periods) tends to have a greater cohesive strength and a higher $\kappa_{0}$.

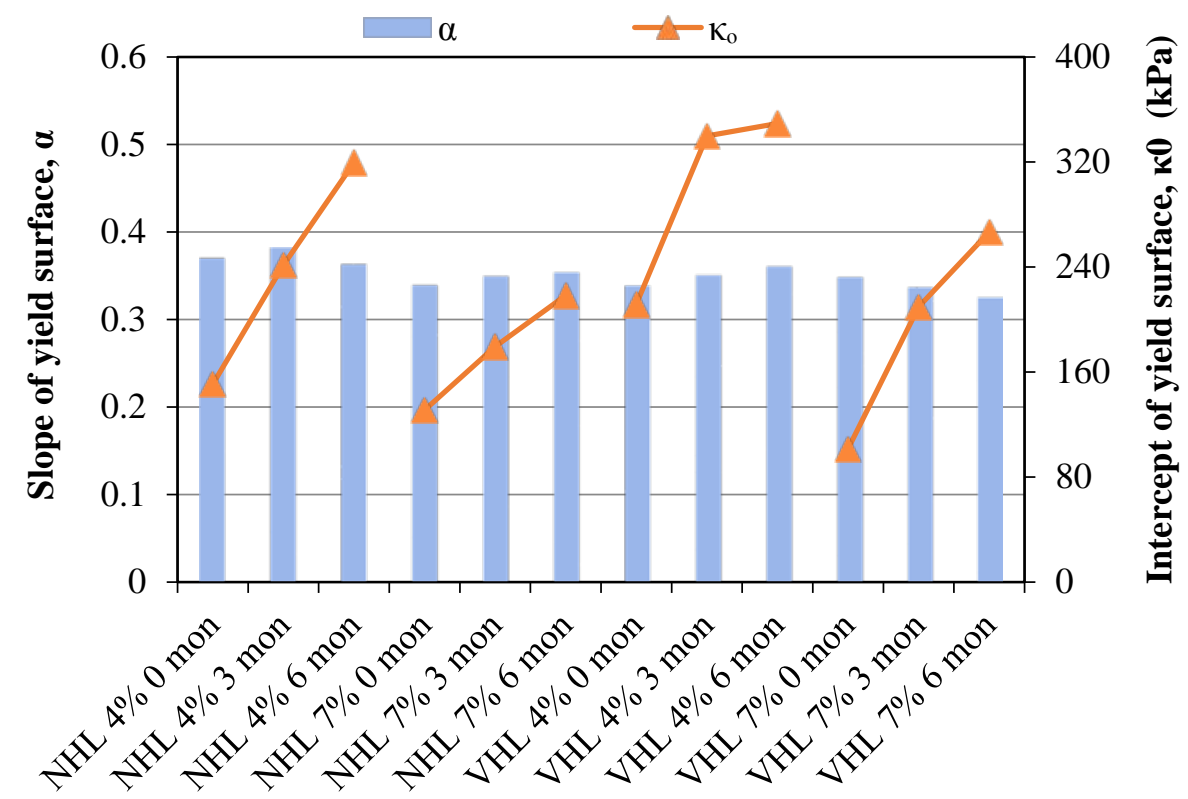

Figure 4. Slope and Intercept of the GD-P Yield Surface on Meridian Plane for Different Asphalt Mixtures (NHL = NuStar binder $($ PG67-22) + Hanson Limestone VHL $=$ Valero binder (PG64-16) + Hanson Limestone; 4\% = 4\% air void content, 3 mon = 3 month continuous aging at $60^{\circ} \mathrm{C}$ ).

Figure 5 shows the hardening parameters $\kappa_{1}$ and $\kappa_{2}$ for different asphalt mixtures. In general, $\kappa_{1}$ determines the amplitude of the strain hardening and it increases as the air void content decreases or the aging period increases. In fact, $\kappa_{1}$ is also fundamentally related to the cohesive properties of the asphalt mixture and a stiffer asphalt mixture tends to have a greater $\kappa_{1}$. The parameter $\kappa_{2}$ determines the rate of the strain hardening for the asphalt mixture in compression. Testing results do not show obvious differences for $\kappa_{2}$ when asphalt mixtures 
have different binders, air void contents, and aging periods. More test results of the model parameters can be found in Zhang (2012).

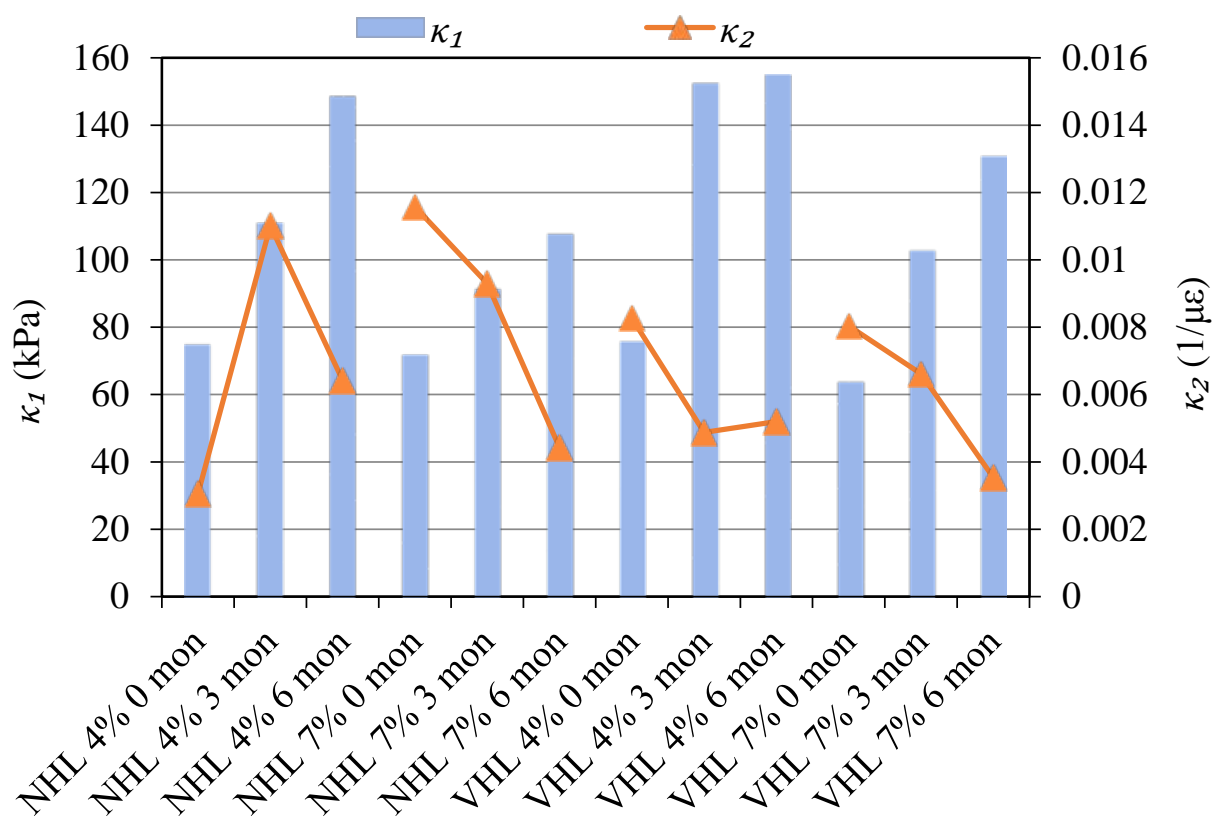

Figure 5. Calculated $\kappa_{1}$ and $\kappa_{2}$ for Different Asphalt Mixtures at $40^{\circ} \mathrm{C}$

\subsection{Viscoelastic-plastic Fracture Constitutive Models}

With small deformation and no cracking damage assumed, the total strain of an asphalt mixture subjected to an external load is decomposed into viscoelastic and viscoplastic strains.

$$
\varepsilon_{i j}=\varepsilon_{i j}^{v e}+\varepsilon_{i j}^{v p}
$$

where $\varepsilon_{i j}=$ total strain tensor, $\varepsilon_{i j}^{v e}=$ viscoelastic strain tensor, and $\varepsilon_{i j}^{v p}=$ viscoplastic strain tensor. If a solid-like generalized Maxwell (Prony) model is used to model the viscoelastic response, i.e., combining Equation (7) in paper (Lytton et al. 2017) with Equations 5 and 23, the apparent total stress becomes

$$
\begin{aligned}
\sigma_{i j}^{A} & =(1-\xi)^{2}\left[K_{\infty}\left(\varepsilon_{k k}^{A}-\varepsilon_{k k}^{v p A}\right) \delta_{i j}+2 G_{\infty}\left(e_{i j}^{A}-e_{i j}^{v p A}\right)\right] \\
& +(1-\xi) \sum_{m=1}^{M}\left\{K_{m}\left[\left(\varepsilon_{k k}^{A}-\varepsilon_{k k}^{v p A}\right)(1-\xi)-\varepsilon_{k k}^{m \cdot v i}\right] \delta_{i j}+2 G_{m}\left[\left(e_{i j}^{A}-e_{i j}^{v p A}\right)(1-\xi)-e_{i j}^{m \cdot v i}\right]\right\}
\end{aligned}
$$

where $\xi$ is damage density, the evolution of which is different in tension and in compression, which are modelled in the following sections. $\varepsilon_{k k}^{A}$ and $e_{i j}^{A}$ are bulk and deviatoric components of apparent total strain, $\varepsilon_{i j}^{A}$, that is determined as $\varepsilon_{i j}^{A}=\left(u_{i, j}+u_{j, i}\right) / 2$, where $u_{i, j}$ is a spatial displacement gradient component. $\varepsilon_{k k}^{v p A}$ and $e_{i j}^{v p A}$ are bulk and deviatoric components of 
apparent viscoplastic strain, $\varepsilon_{i j}^{v p A}$, that is defined as $\varepsilon_{i j}^{v p A}=\varepsilon_{i j}^{v p} /(1-\xi)$, and $\varepsilon_{i j}^{v p}$ is an integration of Equation 9 over time. $\varepsilon_{k k}^{m \cdot v i}$ and $e_{i j}^{m \cdot v i}$ are bulk and deviatoric components of the effective (undamaged) viscous strain caused by the $m$-th dashpot, which are solved by Equation 8 in a previous paper (Lytton et al., 2017).

\section{Viscoelastic Crack Growth in Tension}

\subsection{Principles of Energy Balance}

The principle of force equilibrium indicates that the "apparent force" is equal to the "true force", which leads to the relationship between apparent stress and true stress as in Equation 5. The energy-based mechanics (EBM) approach states that the "true properties" of an asphalt mixture remain unchanged as the damage occurs. What changes as the damage accumulates are the "apparent properties”. Therefore, the true energy within the intact material must be equal to its counterpart from the apparent measurement. Consequently, the true energy within the intact material must equal the apparent energy within the entire specimen, including the intact material and cracks. For instance, the energy dissipated by the entire specimen is equal to that consumed by the intact material, as shown in Equation 25. The energy recovered during crack growth is equal to the energy redistributed in the vicinity of cracks and crack surfaces, as shown in Equation 28. According to this principle, four balance equations are formulated between the true energy and the apparent energy.

Dissipated strain energy (DSE) balance equation: $D S E^{A}=D S E^{T}$

Recoverable strain energy (RSE) balance equation: $R S E^{A}=R S E^{T}$

Dissipated pseudo strain energy (DPSE) balance equation: $D P S E^{A}=D P S E^{T}$

Recoverable pseudo strain energy (RPSE) balance equation:

$$
\operatorname{RPSE}^{A} V_{m}=\operatorname{RPSE}^{T} V_{m}-\operatorname{RPSE}^{T} V_{r}+\Delta S \gamma
$$

where $A$ denotes the "apparent properties”, $T$ denotes the "true properties", $V_{m}$ is the volume of the asphalt mastic in one layer of the asphalt mixture specimen, whose thickness equals the mean film thickness; $V_{r}$ is the volume of the asphalt mastic that is subjected to a relaxation process and releases RPSE during the crack growth, which is calculated as:

$$
V_{r}=\frac{2}{3} M_{N} \pi^{2} \tilde{c}_{N}^{3}-\frac{2}{3} M_{I} \pi^{2} \tilde{c}_{I}^{3}
$$


where $\tilde{C}_{I}$ and $\tilde{c}_{N}$ are average initial crack size before crack growth and average new crack size after crack growth, respectively; $M_{I}$ and $M_{N}$ are the number of initial cracks and number of new cracks, respectively; $\gamma$ is the surface energy density; and $\Delta S$ is the area of all newly created cracks, which is expressed as:

$$
\Delta S=2 M_{N} \pi \tilde{c}_{N}^{2}-2 M_{I} \pi \tilde{c}^{2}
$$

The DPSE represents the total amount of energy dissipated to develop cracking and permanent deformation. From a mechanical point of view, the DPSE for cracking is formulated as:

$$
D_{C S E} V=R_{C} E^{T} V_{r}-\Delta S \gamma
$$

where $D_{P S E_{C}}$ is the DPSE for cracking, and $V$ is the volume of one layer of the asphalt mixture specimen, calculated by multiplying the entire cross-sectional area by the mean film thickness. Then the DPSE for permanent deformation $\left(D P S E_{P}\right)$ is calculated by subtracting $D P S E_{C}$ from the total DPSE:

$$
D P S E_{p}=D P S E^{T}-D P S E_{c}
$$

Details of these energy balance equations can be found in Luo et al. (2013b, 2013e, and 2014b). The above force and energy balance equations are successfully used to determine the true stress and damage density of an asphalt mixture, which is subjected to the repeated loads. As noted above, damage density is represented by a mean size of cracks and the number of such cracks that make up the total lost area. In a uniaxial test, the force and pseudo strain energy balance equations are three simultaneous equations. There are three unknowns in these equations: true stress, mean crack size, and the number of such cracks. In two dimensions, there are total of six of these equations, and in three dimensions, there are nine.

\subsection{Crack Initiation and Endurance Limit in Tension}

The fracture behavior of an asphalt mixture is dependent on the loading level. With the increase of the loading level, the asphalt mixture sequentially experiences the linear viscoelastic state (i.e., line OA in Figure 6), the nonlinear viscoelastic state (i.e., line AB), damage (i.e., line BC), and failure (i.e., line CD), which is illustrated in Figure 6. It has been proven that asphalt mixtures are not damaged when they are under linear and nonlinear viscoelastic states (Luo et al. 2013a). Beyond Point B, the asphalt mixture experiences a 
crack propagation stage until failure. Therefore, the Point B is the threshold between the undamaged and damaged states, where the crack initiation occurs in an asphalt mixture. This threshold (Point B) is called the critical condition for crack initiation or the endurance limit. The material recovery will be complete if an unloading starts before or on Point B, e.g., line BKO. In comparison, the recovery (e.g., line $\mathrm{MN}$ ) will be incomplete if after Point $\mathrm{B}$ and a permanent deformation will be left over.

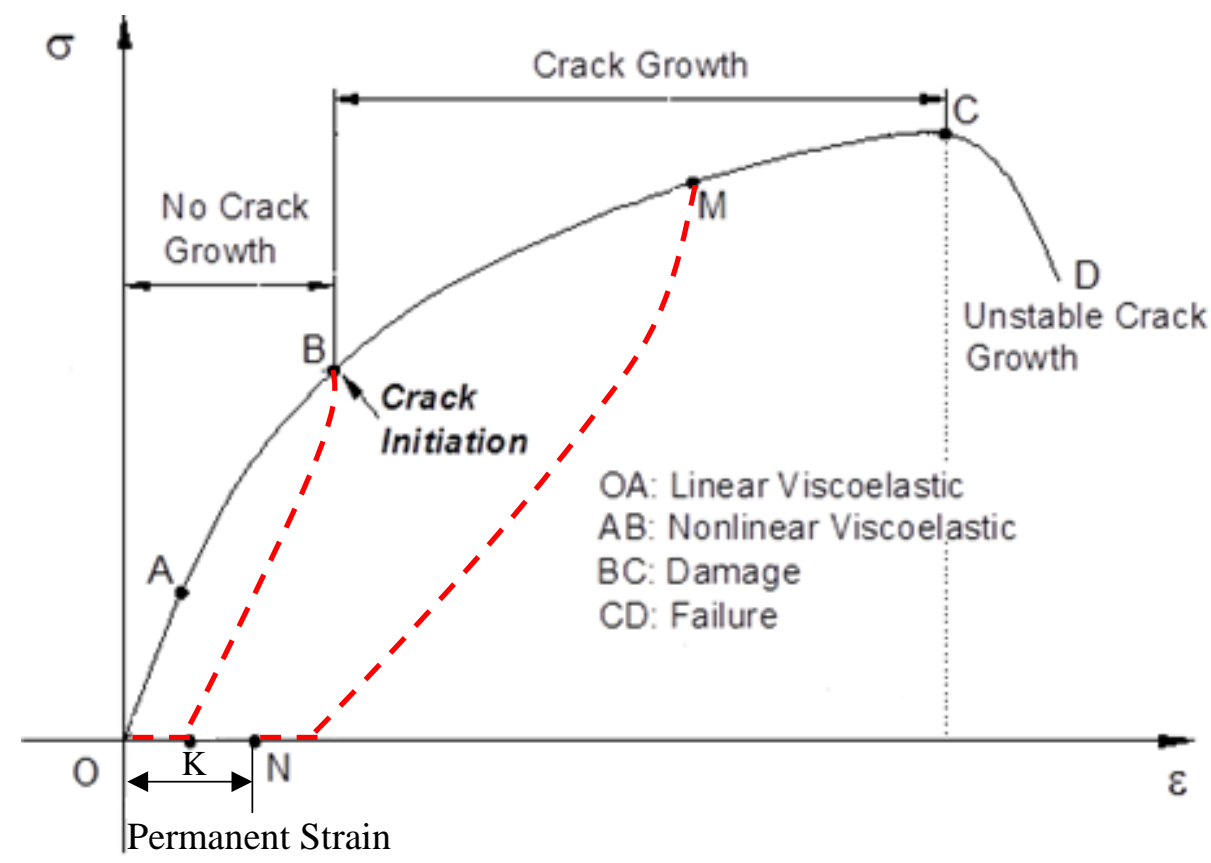

Figure 6. Fracture Behavior of Typical Asphalt Mixtures at Different Loading Levels

In the laboratory, the endurance limit of asphalt mixtures can be identified using the controlled-strain repeated direct tension (RDT) test. A series of controlled-strain RDT tests are performed on the same asphalt mixture specimen at $1 \mathrm{~Hz}$ for 200 loading cycles at incremental strain levels. The first strain level is selected to be $40 \mu \varepsilon$, in which the asphalt mixture experiences a linear viscoelastic stage at room temperature (Gibson et al. 2003). The increment of strain level is $10 \mu \varepsilon$. To ensure that the asphalt mixture fully recovers the deformation, the rest period between the two consecutive tests is designed to be 15 minutes. As shown in Figure 3 of the paper (Lytton et al., 2017), in an undamaged state, the material properties of an asphalt mixture (e.g., complex modulus and phase angle) should remain unchanged as the load cycles increase; in a damaged state, the material properties of an asphalt mixture should vary with the number of load cycles (Luo et al. 2013b). Based on these criteria, the measured material properties from RDT tests are analyzed using a statistical method to examine whether the asphalt mixture specimen is in the undamaged state or damaged state. At the end of the controlled-strain RDT tests, the endurance limit of an 
asphalt mixture can be determined. Table 1 lists the endurance limits of the different asphalt mixtures. It is shown that the endurance limit decreases from unaged asphalt mixtures to those aged for 3 months. This is because the aged asphalt mixtures are more brittle and susceptible to cracking.

Table 1. Endurance Limits of Different Asphalt Mixtures

\begin{tabular}{|c|c|c|c|}
\hline Binder Type & Air Void (\%) & Aging Time (Month) & Endurance Limit $(\boldsymbol{\mu \varepsilon})$ \\
\hline \multirow{3}{*}{ NuStar } & \multirow{2}{*}{$4 \%$} & 0 & 60 \\
\cline { 2 - 4 } & \multirow{2}{*}{$7 \%$} & 3 & 58 \\
\cline { 2 - 4 } & \multirow{2}{*}{$4 \%$} & 0 & 65 \\
\cline { 2 - 4 } & \multirow{3}{*}{ Valero } & 3 & 57 \\
\cline { 2 - 4 } & \multirow{2}{*}{$7 \%$} & 0 & 63 \\
\cline { 2 - 4 } & & 3 & 57 \\
\cline { 2 - 4 } & & 3 & 55 \\
\hline
\end{tabular}

Griffith's crack initiation theory states that the critical condition for crack initiation of an elastic material is an equilibrium condition, where the net change of the potential energy is zero (Griffith 1921). Since an asphalt mixture is a viscoelastoplastic material, the critical condition for crack initiation is expressed as,

$$
\frac{d\left[W_{r}^{R}-\left(W_{n}^{R}+W_{s}\right)\right]}{d A_{s}}=0
$$

where $W_{r}^{R}$ is the pseudo energy released in the local energy release zone, $W_{n}^{R}$ is the pseudo energy consumed in the local nonlinear zone, $W_{s}$ is the surface energy required to create two crack surfaces, and $A_{s}$ is the crack surface area. A detailed form of the energy-based crack initiation criterion for asphalt mixtures in tension is presented in Equation 34.

$$
w_{r}^{R} \pi c_{0}-\frac{9}{8} w_{n}^{R} \pi c_{0}-2 w_{s}=0
$$

where $w_{r}^{R}$ is the released pseudo energy per unit volume in the local energy release zone, $w_{n}^{R}$ is the consumed or dissipated pseudo strain energy in the local nonlinear zone, $w_{s}$ is the surface energy per unit area, and $c_{0}$ is the average air void size. The detailed derivation and validation of the energy-based crack initiation criterion can be found in Luo et al. (2014a). 


\subsection{Viscoelastic Crack Growth Criterion in Tension}

The crack growth of an asphalt mixture in tension has three phases: formative phase, coalescent phase, and unitary phase, which are illustrated in Figure 7. The formative phase starts with the initial air voids as the pre-flaws, from which the microcracks are generated. As the size of microcracks increases to a specific value, the microcracks begin to merge and coalesce with each other. Thereby, the number of microcracks begin to decrease in the coalescent phase. Finally, when a number of microcracks has coalesced into a single macrocrack, the crack growth enters the unitary phase, which dominates the failure of asphalt mixtures.

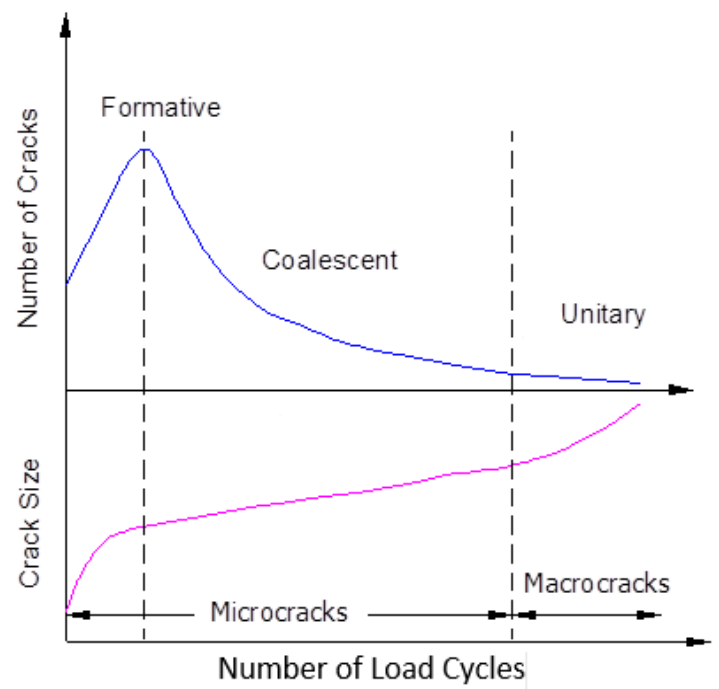

Figure 7. Illustration of Three Phases of Crack Growth in Tension

In the formative and coalescent phases of crack growth, the damage density $\xi$ is expressed as,

$$
\xi=\frac{m \pi c^{-2}}{A}
$$

where $\xi$ is the damage density, $\bar{c}$ is the mean crack size, and $A$ is the cross-sectional area of an asphalt mixture. When the crack growth is in the unitary phase, the damage density is defined as,

$$
\xi=\frac{\pi \bar{c}^{-2}}{A}
$$

Using the controlled-strain RDT test, the damage density of an asphalt mixture is calculated by, 


$$
\xi=\xi_{0}+\xi_{\Delta}=\xi_{0}+\left[1-\frac{\left(\sigma_{0 t}+\sigma_{0 c}\right) / 2}{\sigma_{0}^{T}}\right] V_{m} \%
$$

where $\xi_{0}$ is the initial damage density, which equals the initial air void content of asphalt mixtures, $\xi_{\Delta}$ is the increased damage density due to crack growth, $\sigma_{0 t}$ is the tensile stress amplitude in RDT tests, $\sigma_{0 c}$ is the compressive stress amplitude in RDT tests, $\sigma_{0}^{T}$ is the true stress amplitude solved from the DPSE balance Equation 27, and $V_{m} \%$ is the volumetric percentage of the asphalt mastic that is determined from the volumetric analysis and aggregate gradation of the mixture design.

The crack growth of an asphalt mixture is governed by a modified form of Paris' law (Paris and Erdogan 1963; Lytton et al. 1993), which is expressed as,

$$
\frac{d \xi}{d N}=A^{\prime}\left(\Delta J_{R}\right)^{n^{\prime}}=A^{\prime}\left[\frac{\frac{\partial D P S E}{\partial N}}{\frac{\partial(\text { c.S.a })}{\partial N}}\right]^{n^{\prime}}
$$

where $N$ is the number of load cycles; $\Delta J_{R}$ is the pseudo J-integral, c.s.a is the crack surface area, and $A^{\prime}$ and $n^{\prime}$ are the modified Paris’ law parameters. A sensitivity analysis was conducted on the damage density of asphalt mixtures with various groups of $A^{\prime}$ and $n^{\prime}$ (Luo et al. 2013d). It was found that the value of $n^{\prime}$ is directly related to the resulting damage density. The higher $n^{\prime}$ value results in a higher cracking growth speed. Figure 8 shows the effect of aging time and air void content on fatigue cracking as related to $n '$. The value of $n^{\prime}$ increases with the aging time, which indicates that the aged asphalt mixture has less resistance to fatigue cracking damage. It is also shown that the asphalt mixtures with $7 \%$ air void content have higher $n^{\prime}$ values than those with $4 \%$ air void content. This demonstrates that the asphalt mixtures with $7 \%$ air void content are more susceptible to fatigue cracking than those with $4 \%$ air void content. 


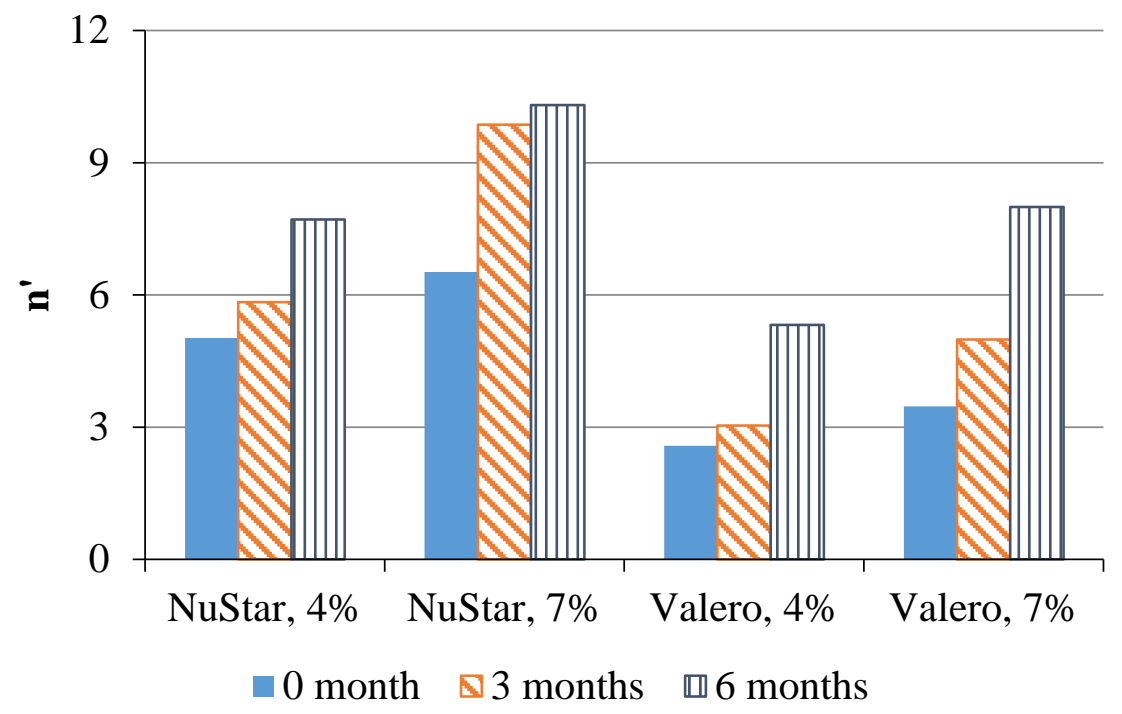

Figure 8. Effect of Aging Time and Air Void Content on Fatigue Cracking Speed of

\section{Asphalt Mixtures}

Figures 9a and 9b present the relationship between fracture coefficients $A^{\prime}$ and $n^{\prime}$ for laboratory-compacted asphalt mixtures and field cores, respectively. A linear relationship is found between $-\log A^{\prime}$ and $n^{\prime}$ for both lab-compacted asphalt mixtures and field cores (Jacobs 1995; Luo et al. 2013d; Gu et al. 2014; Gu et al. 2015).

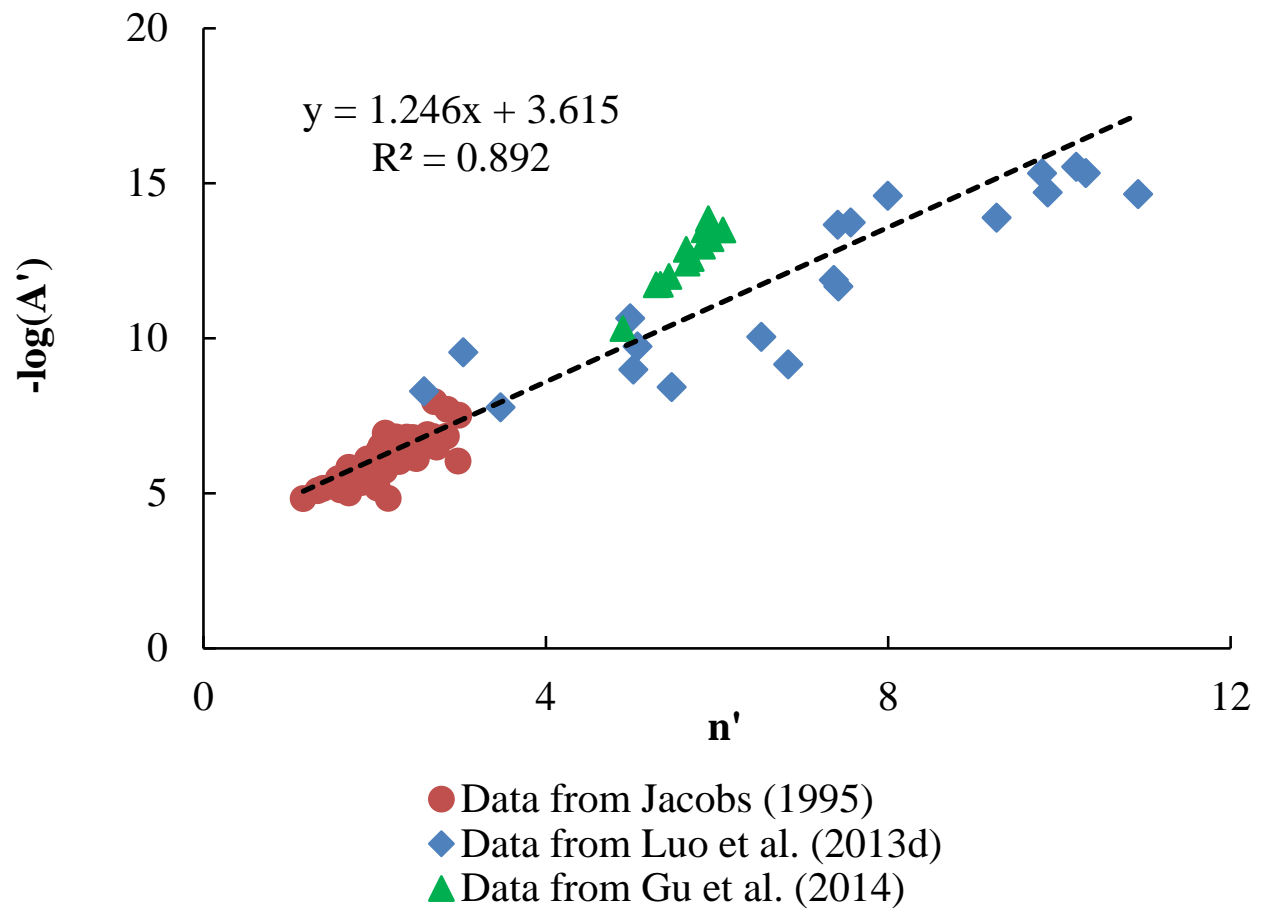

a. Relationship between $A^{\prime}$ and $n^{\prime}$ for Laboratory-Compacted Asphalt Mixtures 


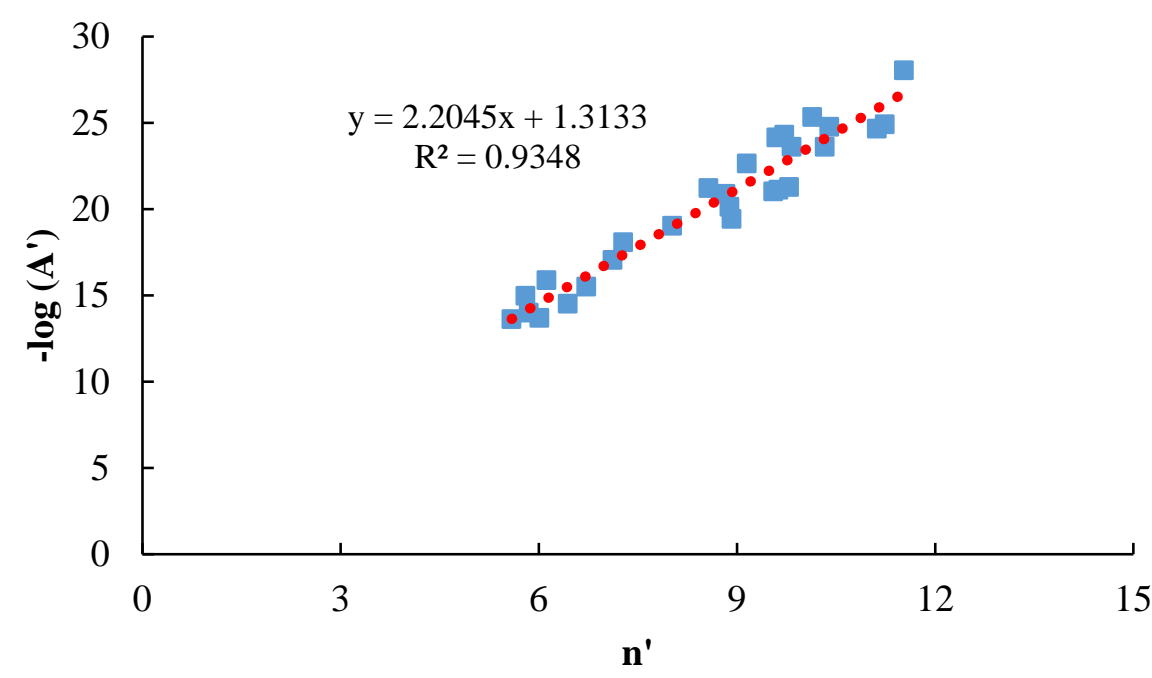

b. Relationship between $A^{\prime}$ and $n^{\prime}$ for Asphalt Field Cores

Figure 9. Relationship between Paris' Law Coefficients $A^{\prime}$ and $n^{\prime}$ for Crack Evolution of Asphalt Mixtures

\section{Viscoelastic Crack Growth in Compression}

Field and laboratory observations have shown that cracks accompany rutting in asphalt layers when subjected to external compressive loads (Wang et al., 2003; Underwood et al., 2011). Mechanical analyses demonstrate that the overstress softening behavior under a monotonic compressive load and the tertiary flow of rutting under a repeated compressive load result from cracking together with plastic deformation (Zhang et al. 2014b; Zhang et al. 2012).

\subsection{Viscofracture Crack Initiation in Compressive Loading}

Cracks in compression are inclined to grow toward (not the same as) the direction of the external compressive loads. This differs from that in tension cracks which grow in the direction perpendicular to the external tensile loads. The wing cracks are where the new cracks grow in compression, as shown in Figure 10. They are driven by the localized tensile or shear stresses near the tips of and parallel to the pre-existing cracks which are the compressed air voids in asphalt mixtures. 


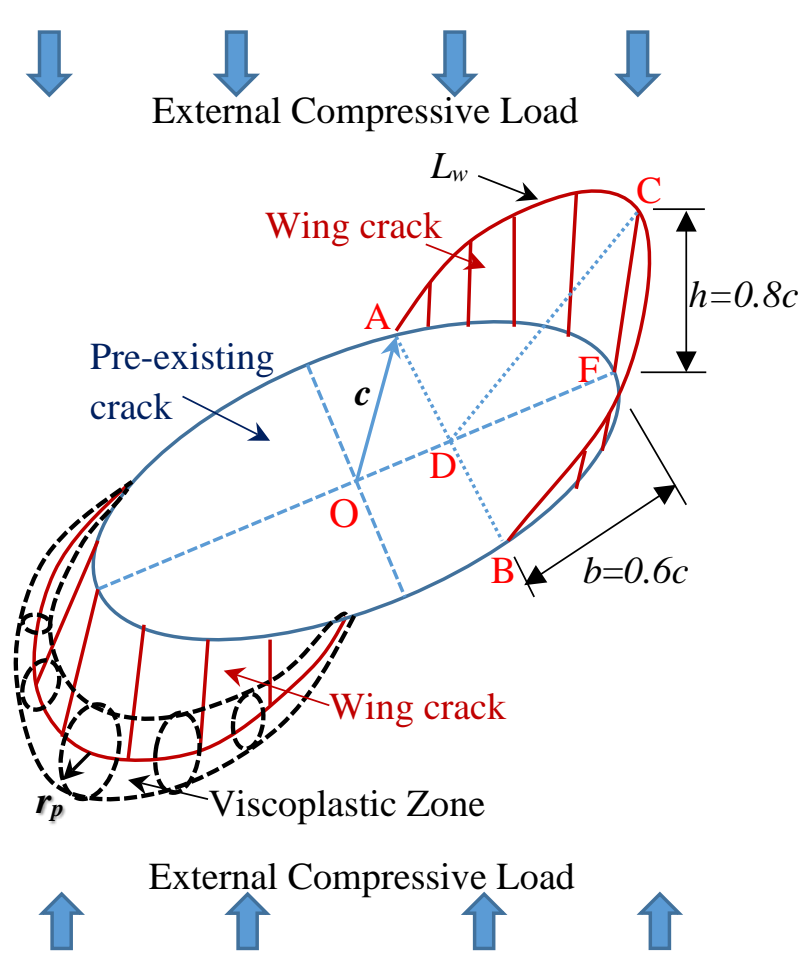

Figure 10. Configurations of a Pre-existing Crack and Propagated Wing Cracks under an External Compressive Load in Vertical Direction

The crack (damage) initiation in compression is modelled by the viscoelastic Griffith theory and cracks will grow when the below energy equation is satisfied (Zhang et al. 2014c):

$$
\text { RPSE }-\frac{1}{2} \text { DPSE }=\frac{7}{6} \pi \frac{\Delta G}{c}
$$

where RPSE $=\left(\sigma_{11}^{2}+\sigma_{22}{ }^{2}+\sigma_{33}^{2}\right) /\left(2 E_{R}\right)+2\left(1+v_{R}\right)\left(\sigma_{12}^{2}+\sigma_{23}{ }^{2}+\sigma_{13}{ }^{2}\right) / E_{R}=$ recoverable pseudo strain energy (density), $E_{R}$, and $v_{R}=$ reference modulus and Poisson's ratio which are derived to be Young's modulus and elastic Poisson's ratio (Zhang et al. 2012). DPSE = dissipated pseudo strain energy (density) due to the accumulation of the viscoplastic deformation, and $D P S E=\int_{0}^{t} \sigma_{i j} \dot{\varepsilon}_{i j}^{v p} d t . c=$ average air void radius that is also the critical crack size at which a crack starts to grow. This critical crack size is related to the air void content of a mixture (Zhang et al. 2014c):

$$
c=0.0037(\% A V)^{2}+0.0071(\% A V)+0.5583 \quad R^{2}=0.7431
$$

where $\% A V=$ air void content of an asphalt mixture in percentage. $\Delta G=$ bond energy of the asphalt mixture, which represents a combined bond energy for cohesive fracture and adhesive fracture. A higher temperature induces a greater percentage of cohesive fracture and therefore leads to a lower bond energy (Zhang et al. 2014c). The temperature dependent bond energy is modelled as: 


$$
\begin{aligned}
& \Delta G(T)=a_{G} \Delta G\left(T_{r}\right) \\
& a_{G}(T)=\exp \left[\frac{\Delta E_{G}}{R}\left(\frac{1}{T}-\frac{1}{T_{r}}\right)\right]
\end{aligned}
$$

where $a_{G}=$ temperature shift factor for bond energy; $\Delta E_{G}=$ activation energy for the temperature effect on bond energy.

\subsection{Viscofracture Crack Evolution in Compressive Loading}

The crack damage evolution follows a pseudo J-integral Paris' law (Zhang et al. 2013b;

Zhang et al. 2014b; Zhang et al. 2016). In a monotonic compressive load test, one can derive that

$$
\frac{d \xi}{d t}=A\left(\Delta J_{R}\right)^{n}=A\left[\frac{H}{2} \frac{(3+\xi)}{(1-\xi)^{3}} R P S E\right]^{n}
$$

where $d \xi / d t=$ rate of damage density; $A$ and $n=$ Paris' law coefficients that are demonstrated to be independent of loading mode, rate, and temperature (Zhang et al., 2016); $\Delta J_{R}=$ pseudo J-integral per loading step; $H=$ height of a laboratory sample or thickness of an asphalt layer in the pavement.

In a repeated compressive load test, using an incremental dissipated pseudo strain energy balance principle for viscofracture, the axial damage density is given by:

$$
\xi_{1}(N)=1-\frac{\sigma_{0}^{A}}{\sigma_{N}^{T}}=1-\left[\left(\frac{1}{1-\xi_{01}}\right)^{2}+\frac{\Delta \varepsilon_{1}^{v f}(N)}{\sigma_{0}^{A}} \frac{E_{Y}}{\pi \sin \left(\delta-\varphi_{I I}\right)}\right]^{-\frac{1}{2}}
$$

It can be shown from Equation 44 that the axial damage density is determined by the incremental viscofracture strain as well as the fundamental material properties including Young's modulus, phase angle of undamaged asphalt mixtures and phase angle in the secondary deformation stage under a destructive repeated load.

The radial damage density can be derived based on the volume balance principle, which means that the increased volume of the asphalt mixture caused by viscofracture strains should equal the volume of cracks, thus:

$$
\xi_{2}=\frac{\left(1+\varepsilon_{2}^{v f}\right)^{2}\left(1-\varepsilon_{1}^{v f}\right)-1}{\sqrt{\xi_{1}}}
$$


Figure 11 demonstrates that the Paris' law coefficients ( $A$ and $n$ ) for six types of asphalt mixture determined from monotonic load tests are comparable to that from repeated load tests at the same temperatures. It is also found that $A$ decreases and $n$ increases when the asphalt mixture become stiffer which is due to a combination of a stiffer binder, a lower air void content and a longer aging period. This observation complies with Schapery's viscoelastic fracture theory (Schapery 1984; Kuai et al. 2009) as well as the results in the preliminary study (Zhang et al. 2013b). Based on Schapery's theory, $n$ is inversely proportional to the slope of the creep compliance, e.g. $n=1+1 / m$, where $m$ is the slope of the creep compliance that is modeled by $D(t)=D_{0}+D_{2} t^{m}$. It is known that a stiffer asphalt mixture normally has a smaller value of $m$ than a softer asphalt mixture. Thus the stiffer asphalt mixture has a relatively larger $n$ value, which is verified by the testing results of this study. Further comparison of axial and radial damage densities for stiff and soft asphalt mixture (e.g., due to low or high air void contents) indicates that a stiff asphalt mixture tends to have a brittle cracking and a soft asphalt mixture has a ductile cracking (Zhang et al. 2014b).

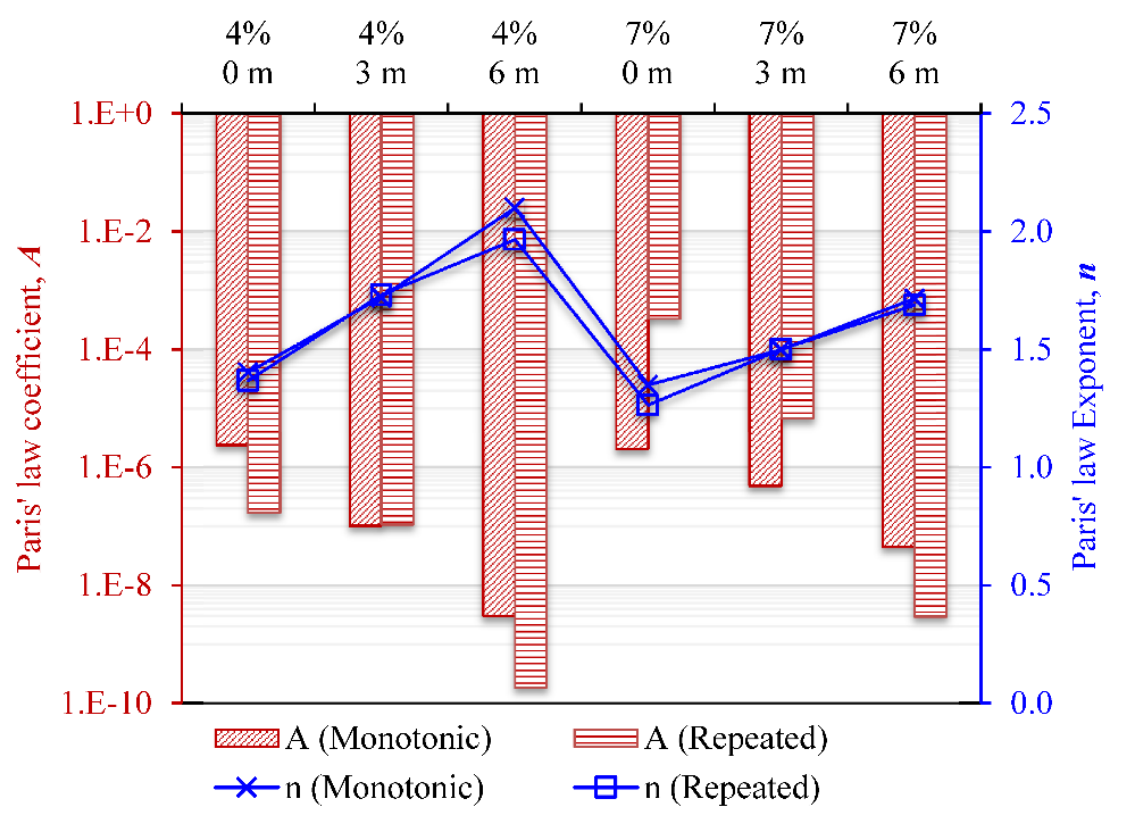

Figure 11. Paris' Law Coefficients for Different Asphalt Mixtures Determined from

Monotonic/Repeated Load Tests (4\% is air void content, $3 \mathrm{~m}$ stands for aged at $60^{\circ} \mathrm{C}$ for 3 months)

Figure 12 shows that the Paris' law coefficients of a specific asphalt mixture do not vary significantly with temperatures or loading rates. Thus the Paris' law coefficients are fundamental material properties and independent of temperature, loading mode or rate. Note 
that this conclusion is valid only when the pseudo J-integral $\left(J_{R}\right)$ is used in Paris' law. It is because $J_{R}\left(J_{R}=\int_{0}^{t} D(t-s) \frac{d K^{2}}{d s} d s\right)$ addresses the temperature effect by creep compliance $(D(t))$ and accounts for loading (mode or rate) effects by the stress intensity factor $(K)$. Since repeated load tests are costly and time consuming, monotonic load tests (e.g., compressive strength tests) are recommended to determine the Paris' law coefficients for an asphalt mixture. Then the coefficients can be implemented in Paris' law using an accurate pseudo Jintegral to predict the crack growth of the asphalt mixture under different loading and temperature conditions. More test data for $\mathrm{A}$ and $\mathrm{n}$ for different asphalt mixtures can be found in Zhang (2012), where strong relationships are found between $\log \left(A_{i}\right)$ and $n_{i}$, similar to that in tension.

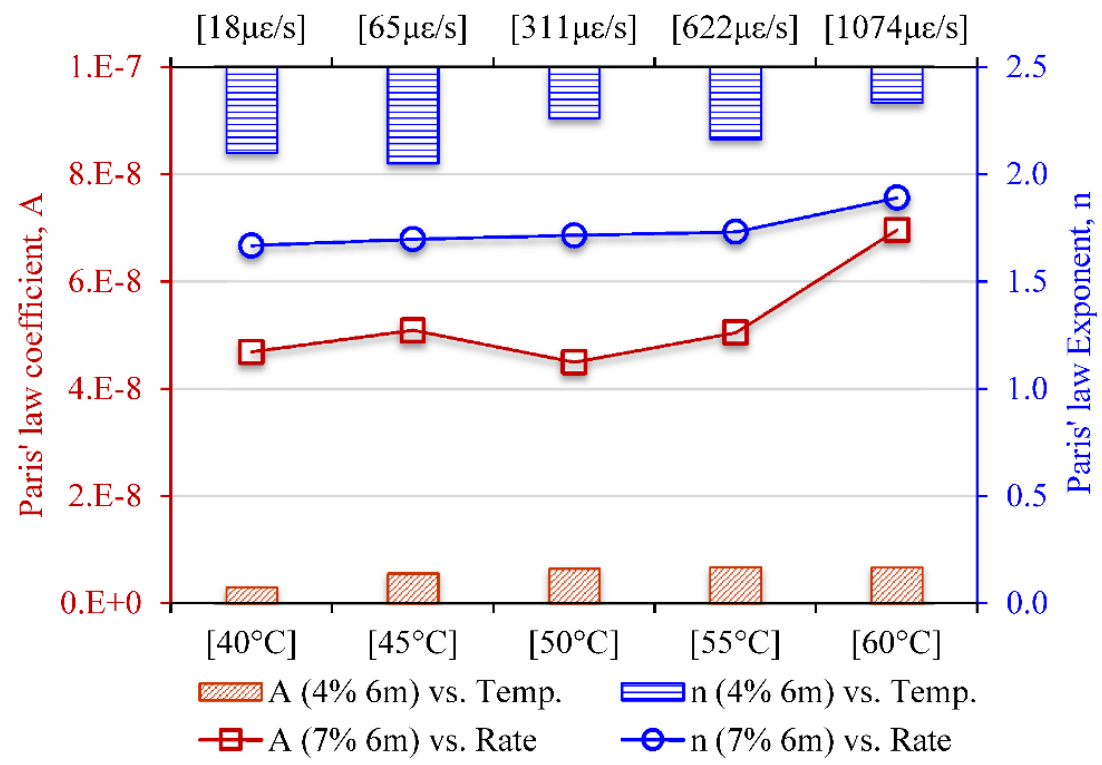

Figure 12. Paris' Law Coefficients of Asphalt Mixtures Tested at Different Temperatures and Loading Rates

\section{Healing of Asphalt Mixtures in Tension}

Healing refers to a process of the restoration of original material properties to a deformed material after the load is removed. For asphalt mixtures, it has been proven that healing is a substantially important issue, and its significance has been demonstrated in both the laboratory and the field (Lytton et al. 1993; Kim et al. 1990; and Williams et al. 1998). An energy-based mechanics approach has been developed to quantify the healing properties of asphalt mixtures, which considers the internal stress is the principal driving force for the healing. A creep and step-loading recovery (CSR) test is designed to directly measure the internal stress during the recovery of the asphalt mixtures, which is further used to 
characterize the healing properties. The Ramberg-Osgood equation is employed to predict the healing behavior of asphalt mixtures.

\subsection{Creep and Step-loading Recovery Test Method}

A schematic plot of a CSR test is shown in Figure 13. In the creep phase, the creep strain is accumulated and the asphalt mixture specimen is stretched from State A to State B. When the load is removed at $t_{0}$, the asphalt mixture specimen begins to recover the deformation. The remaining deformation of the specimen is defined as the residual strain $\varepsilon_{r}$. The recovery process is driven by the internal stress $\sigma_{i}$, which is shown as a group of arrows in States C, $\mathrm{D}$ and $\mathrm{E}$. In the recovery phase, three external step-loads (e.g., $\sigma_{1}, \sigma_{2}$ and $\sigma_{3}$ ) are applied to the specimen.

- If $\sigma_{1}<\sigma_{i}$, the residual strain rate $\dot{\varepsilon}_{r}<0$; and

- If $\sigma_{2}=\sigma_{i}$, the residual strain rate $\dot{\varepsilon}_{r}=0$; and

- If $\sigma_{3}>\sigma_{i}$, the residual strain rate $\dot{\varepsilon}_{r}>0$

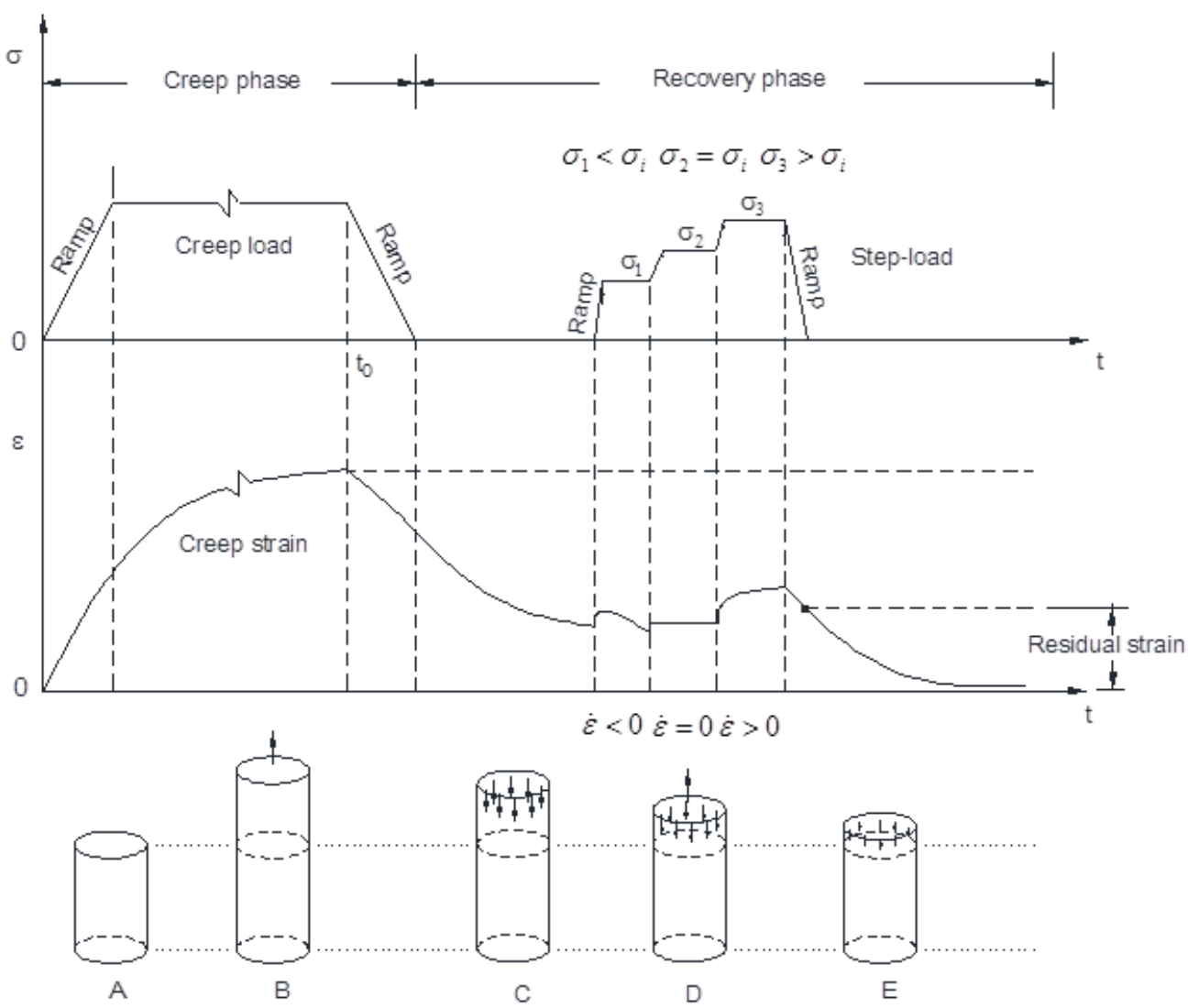

Figure 13. Schematic Plot of Creep and Step-Loading Recovery Test 
A group of step-load trials are designed to detect the state in which the residual strain rate equals zero. In this way, the internal stress of an asphalt mixture specimen is measured by the CSR test. The measured internal stress is used to define a new type of material property: the recovery modulus, which is an indicator to the recovery behavior of asphalt mixtures. The recovery modulus of asphalt mixture is calculated by,

$$
R(t)=\frac{\sigma_{i}(t)}{\varepsilon_{r}(t)}
$$

where $R(t)$ is the recovery modulus of the asphalt mixture, $\sigma_{i}(t)$ is the internal stress obtained from the CSR test, and $\varepsilon_{r}(t)$ is the residual strain in the recovery phase.

To characterize the healing properties of asphalt mixtures, the CSR test includes a nondestructive stage and a destructive stage, which is detailed in Luo et al. (2013c). Figure 14 compares the recovery modulus of an asphalt mixture in the nondestructive stage and destructive stage. The recovery modulus in the nondestructive stage does not change as the loading level increases. This indicates that the recovery behavior of undamaged asphalt mixtures is only dependent on the material itself. The recovery modulus in the destructive stage is smaller than that in the nondestructive test. This is explained as the loss by the damaged asphalt mixture of part of its capability to recover.

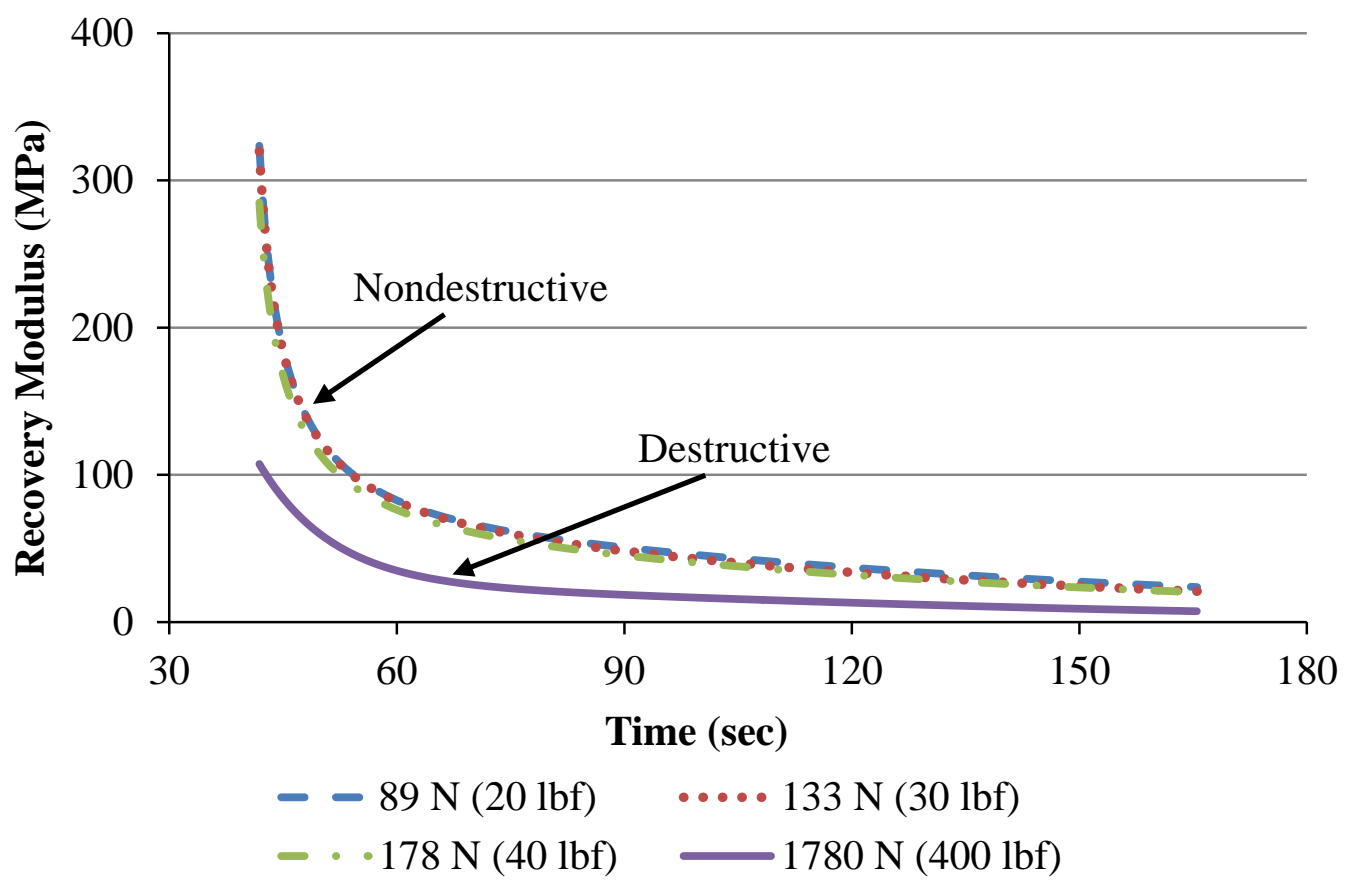

Figure 14. Recovery Modulus of Asphalt Mixtures in Nondestructive and Destructive CSR Test 


\subsection{Mechanistic Modeling of Healing in Asphalt Mixture}

The healing behavior is quantified by the normalized extent of healing, which is defined as,

$$
h=\frac{\xi_{f c}-\xi_{i}}{\xi_{f c}-\xi_{0}}, h \in[0,100 \%]
$$

where $h$ is the normalized extent of healing, $\xi_{f c}$ is the final damage density at the end of the creep phase, $\xi_{i}$ is the damage density at any data point $i$ of the recovery phase, $\xi_{0}$ is the initial damage density. The EBM approach is used to determine the damage density of an asphalt mixture in the creep phase and recovery phase. The major steps are summarized as follows.

- Determine apparent and true material properties of undamaged asphalt mixtures;

- Calculate pseudo strain in the creep phase;

- Calculate DPSE using pseudo strain for damaged asphalt mixtures;

- Determine true creep strain, true creep stress, and damage density in the creep phase;

- Calculate pseudo strain in the recovery phase;

- Calculate RPSE using pseudo strain for damaged asphalt mixtures;

- Determine damage density of asphalt mixtures in the recovery phase.

Figure 15a presents an example of a healing curve (the normalized extent of healing versus the rest time) of an asphalt mixture. It is shown that a rapid increase of the measured healing occurs in the first 10 seconds. After that, the healing rate gradually decreases as the

rest time increases. In Figure $15 \mathrm{a}, \dot{h}_{1}$ is the initial healing rate, representing the healing speed of the material at the initial stage of healing; $\dot{h}_{2}$ is the ultimate healing rate, indicating the healing speed of the material after a long time, and $h_{\beta}$ is the healing scale, reflecting the overall ability of the material to heal. Figure 15b indicates that the healing curves of asphalt mixtures are influenced by the binder type and air void content. The healing curve decreases when the air void content increases from $4 \%$ to $7 \%$ for asphalt mixtures with both AAD and AAM binders. Figure 15c shows the effect of temperature and aging time on the healing curves of asphalt mixtures. The healing of an asphalt mixture increases with the temperature, and decreases with the aging time. The Ramberg-Osgood equation is used to predict the healing of asphalt mixtures, which is shown in Equation 48. 


$$
\hat{h}=\dot{h}_{2} \Delta t+\frac{\left(\dot{h}_{1}-\dot{h}_{2}\right) \Delta t}{1+\frac{\left(\dot{h}_{1}-\dot{h}_{2}\right) \Delta t}{h_{\beta}}} h_{\beta}
$$

where $\hat{h}$ is the predicted healing based on these three parameters: $\dot{h}_{1}, \dot{h}_{2}$ and $h_{\beta}$; and $\Delta t$ is the rest time. The three healing parameters $\dot{h}_{1}, \dot{h}_{2}$ and $h_{\beta}$ are dependent on the undamaged properties of asphalt mixtures, which are formulated as,

$$
\begin{aligned}
& \dot{h}_{1}=a_{1}\left(\frac{1}{\Delta G^{L W} E_{1}}\right)^{b_{1} \kappa} \\
& -\log \left(\dot{h}_{2}\right)=a_{2}\left[-\log \left(\frac{\Delta G^{A B}}{E_{1}}\right)\right]^{b_{2} \kappa} \\
& h_{\beta}=a_{\beta}\left(\frac{\Delta G^{A B}}{\Delta G^{L W} E_{1}^{2}}\right)^{\left(1-h_{0}\right) b_{\beta} \kappa}
\end{aligned}
$$

where $\Delta G^{L W}$ is the non-polar surface bond energy; $\Delta G^{A B}$ is the polar surface bond energy; $E_{1}$ and $\kappa$ are the parameters for the relaxation modulus of undamaged asphalt mixtures; $h_{0}$ is the initial damage density; and $a_{1}, b_{1}, a_{2}, b_{2}, a_{\beta}, b_{\beta}$ are the fitting parameters.

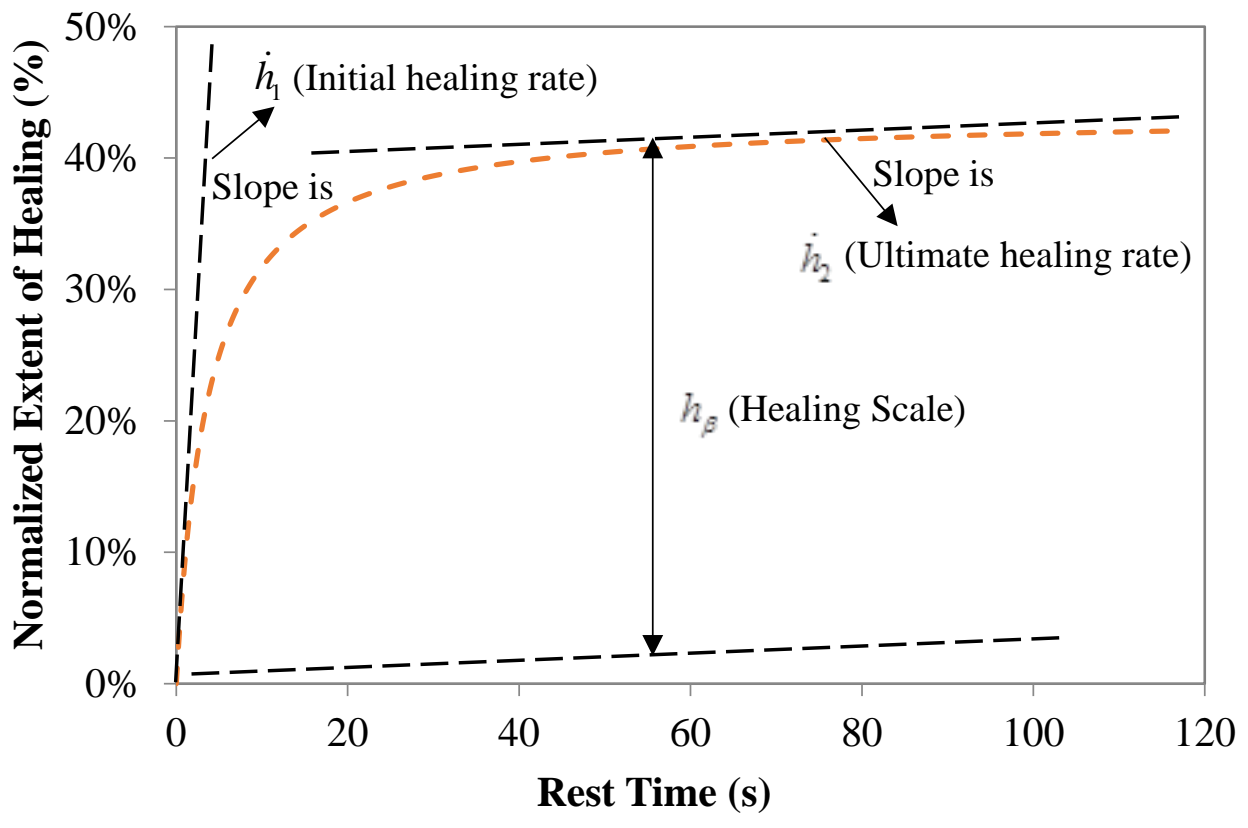

a. Illustration of Healing Curve of Asphalt Mixture 


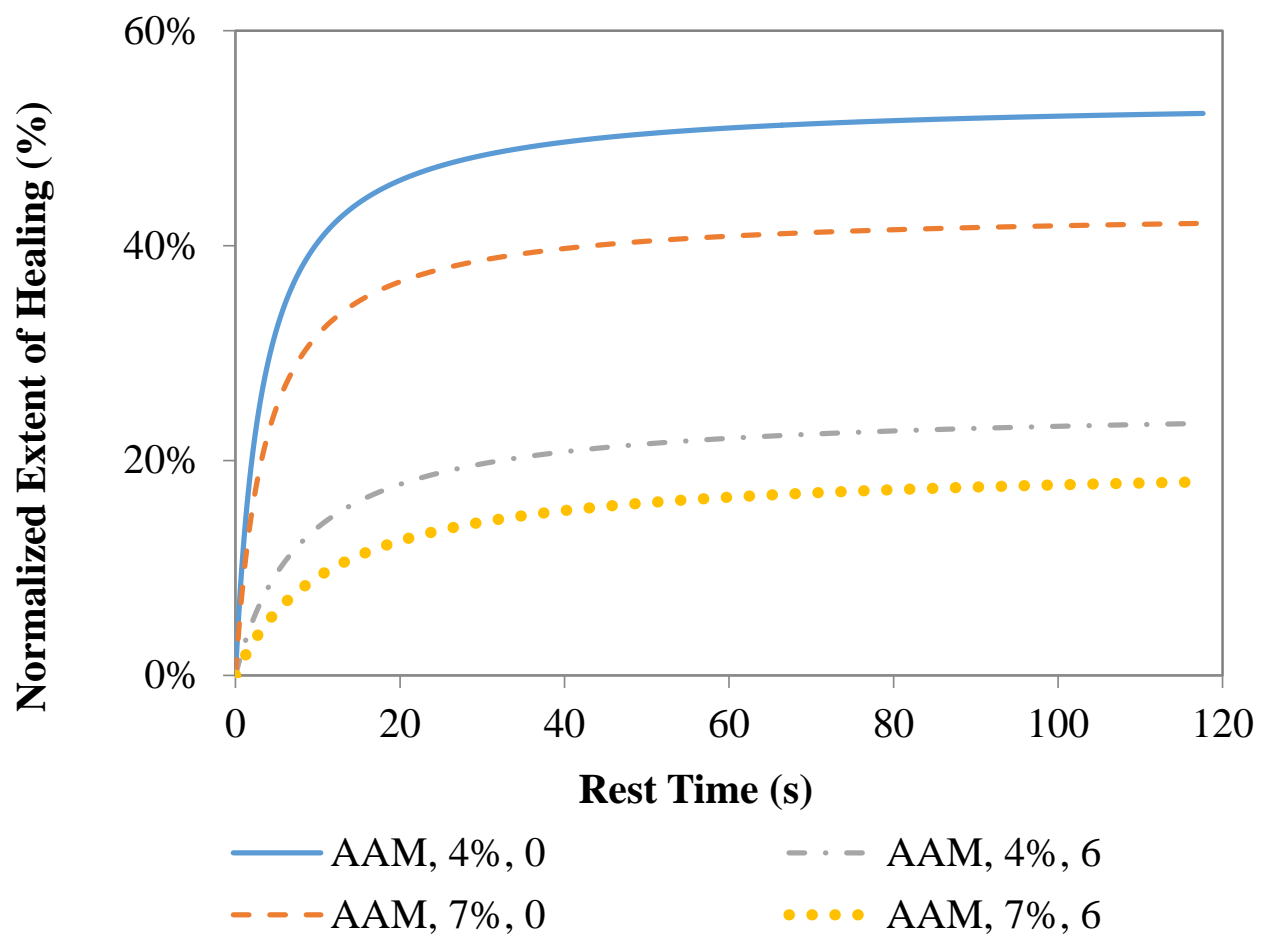

b. Effect of Binder Type and Air Voids on Healing Curves of Asphalt Mixtures

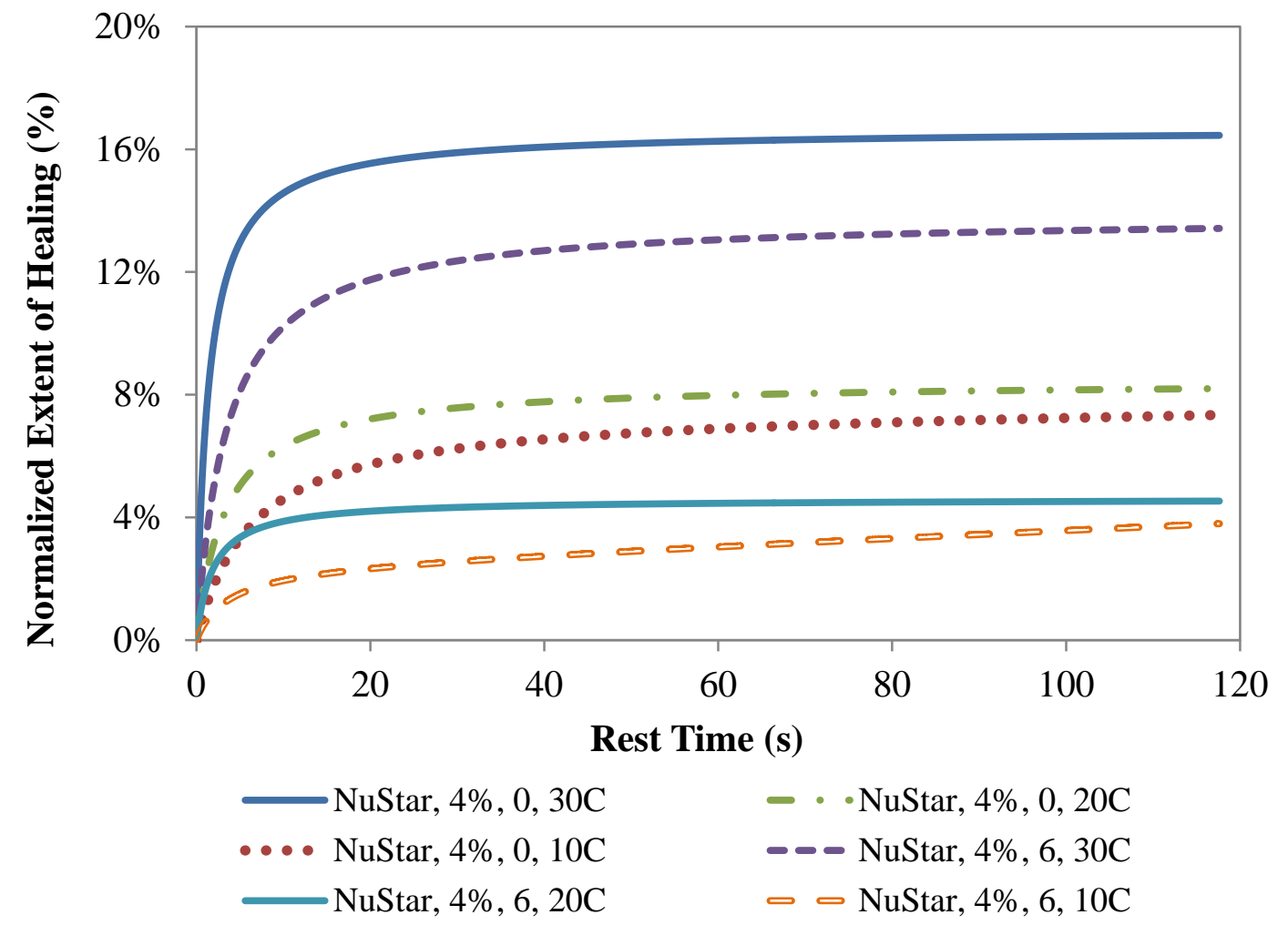

c. Effect of Temperature and Aging Time on Healing Curves of Asphalt Mixtures

Figure 15. Example of Healing Curves of Asphalt Mixtures 


\section{Damage Characterization Tests for Asphalt Mixtures}

\subsection{Characterization Tests under Tensile Loading}

Table 2 summarizes the test methods, the corresponding test time, and damaged material properties of asphalt mixtures under tensile loading. The RDT test with non-destructive and destructive stages is used to characterize the fatigue cracking properties of asphalt mixtures. The determined fatigue cracking properties of asphalt mixtures include the complex modulus and phase angle, the endurance limit, the pseudo strain, the evolution of DPSE, RPSE and damage density, and the two Paris’ Law coefficients A and n. The CSR test with nondestructive and destructive stages is used to characterize the healing properties. The determined healing properties include the internal stress, the recovery modulus, and the three healing parameters $\dot{h}_{1}, \dot{h}_{2}$ and $h_{\beta}$. All of the damaged properties of asphalt mixtures can be measured simply, accurately and quickly.

Table 2. Summary of Test Methods, Test Time and Damaged Material Properties of Asphalt Mixtures in Tension

\begin{tabular}{|c|c|c|c|}
\hline $\begin{array}{c}\text { Material } \\
\text { Properties }\end{array}$ & Test Methods & Test Time & $\begin{array}{c}\text { Damaged Model } \\
\text { Parameters }\end{array}$ \\
\hline \multirow{11}{*}{ Fatigue Cracking } & \multirow{11}{*}{$\begin{array}{c}\text { Repeated Direct } \\
\text { Tension (RDT) Test: } \\
\text { Non-destructive \& } \\
\text { Destructive }\end{array}$} & \multirow{11}{*}{ Less Than 2 Hours } & Complex Modulus E $^{*}$ \\
\hline & & & Phase Angle $\varphi$ \\
\hline & & & Endurance Limit $\varepsilon$ \\
\hline & & & Pseudo strain $\varepsilon^{R}$ \\
\hline & & & DPSE \\
\hline & & & RPSE \\
\hline & & & Mean Crack Size $c_{i}$ \\
\hline & & & Number of Cracks $M_{i}$ \\
\hline & & & Damage Density $\xi$ \\
\hline & & & $\begin{array}{c}\text { Paris' Law Coefficient } \\
A\end{array}$ \\
\hline & & & $\begin{array}{c}\text { Paris’ Law Coefficient } \\
n\end{array}$ \\
\hline \multirow{5}{*}{ Healing } & \multirow{5}{*}{$\begin{array}{l}\text { Creep and Step- } \\
\text { Loading Recovery } \\
\text { Test: Non-destructive } \\
\text { and Destructive }\end{array}$} & \multirow{5}{*}{ Less Than 3 Hours } & Internal Stress $\sigma_{i}$ \\
\hline & & & $\begin{array}{c}\text { Recovery Modulus } \\
R(t)\end{array}$ \\
\hline & & & Initial Healing Rate $\dot{h}_{1}$ \\
\hline & & & $\begin{array}{l}\text { Ultimate Healing Rate } \\
\qquad \dot{h}_{2}\end{array}$ \\
\hline & & & Healing Scale $h_{\beta}$ \\
\hline
\end{tabular}




\subsection{Characterization Tests under Compressive Loading}

Table 3 summarizes the test methods, testing parameters, material properties and the corresponding references where the data were reported for the damage characterization of four different types of asphalt mixtures in compression. Note that the undamaged characterization including viscoelastic properties such as dynamic modulus master curves and time-temperature shift factor have been discussed in the paper (Lytton et al., 2017). Lateral surface scanning tests were used to determine the anisotropic vector magnitude $\left(\Delta^{\prime}\right)$ so that the slope of the plastic potential $(\beta)$ can be calculated using Equation 22 (Zhang 2012). The viscoplastic yield surface and strain hardening parameters were determined from the constant-strain-rate compressive strength (CS) tests (using the stress-strain curve before peak stress) at different confinements and temperatures (Zhang et al. 2013a). The Perzyna's parameters were determined by destructive repeated compressive load (RL) tests to evaluate the viscosity and loading rate dependence (Zhang 2012). The bond energy $(\Delta G)$ can be backcalculated from compressive strength based on the CS test or estimated by cohesive and adhesive surface energy components measured by surface energy tests (Zhang et al. 2014c). The Paris' law coefficients for viscofracture models were proven to be independent of temperature, loading mode and rate, which were identified by the overstress part (after the peak stress) of the CS tests (Zhang et al. 2016; Zhang et al. 2017) or the tertiary flow part of the RL tests (Zhang et al. 2013b).

Table 3. Testing Methods, Asphalt Mixtures, and Material Properties as Model Inputs.

\begin{tabular}{|c|c|c|c|c|c|c|c|}
\hline \multirow{4}{*}{$\begin{array}{l}\text { Material } \\
\text { Property }\end{array}$} & \multirow{3}{*}{ Asphalt Mixtures } & Binder & \multicolumn{4}{|c|}{ Binder-N (PG64-16) } & \multirow{4}{*}{$\begin{array}{c}\text { Data } \\
\text { Resources }\end{array}$} \\
\hline & & Air Void & \multicolumn{3}{|c|}{$4 \%$} & \multirow{2}{*}{ 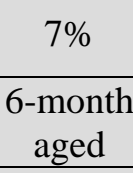 } & \\
\hline & & Aging & Unaged & $\begin{array}{c}\text { 3-month } \\
\text { aged }\end{array}$ & $\begin{array}{c}\text { 6-month } \\
\text { aged }\end{array}$ & & \\
\hline & Test Methods & Label & N4-0 & N4-3 & N4-6 & N7-6 & \\
\hline \multirow{6}{*}{$\begin{array}{c}\text { Visco- } \\
\text { plasticity }\end{array}$} & \multirow{6}{*}{$\begin{array}{c}\text { *Compressive } \\
\text { Strength (CS) } \\
\text { tests @ } 40^{\circ} \mathrm{C} \text {, and } \\
\text { confinements of } \\
0,103.5,207 \mathrm{kPa} \\
\text { (uses }<5 \text { minutes } \\
\text { for each CS test) }\end{array}$} & $\phi\left(^{\circ}\right)$ & 46.8 & 48.9 & 45.9 & 44.8 & \multirow{6}{*}{$\begin{array}{c}\text { Table } 3 \text { of } \\
\text { Ref.(Zhang } \\
\text { et al. } \\
\text { 2013a) }\end{array}$} \\
\hline & & $C(k P a)$ & 143.6 & 227.3 & 302.5 & 203.9 & \\
\hline & & $\alpha$ & 0.370 & 0.382 & 0.363 & 0.354 & \\
\hline & & $\kappa_{0}(k P a)$ & 150.8 & 241.5 & 319.6 & 218.4 & \\
\hline & & $\kappa_{1}(k P a)$ & 75.0 & 111.0 & 148.6 & 107.8 & \\
\hline & & $\kappa_{2}(1 / \varepsilon)$ & 3,060 & 11,010 & 6,410 & 4,430 & \\
\hline
\end{tabular}




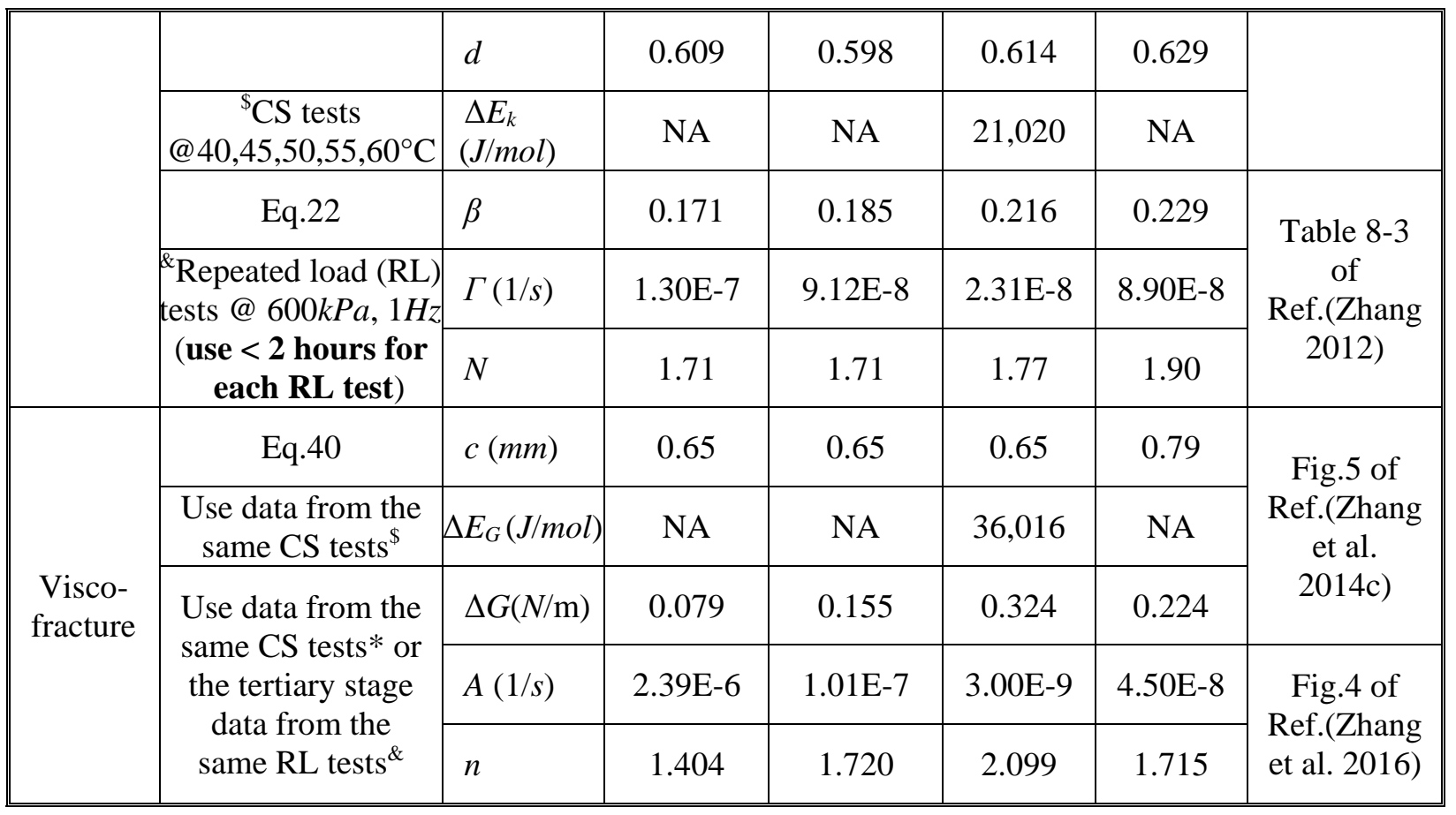

\section{Summary}

This paper has illustrated an energy-based mechanics measuring the material properties that are characteristics of fracture, healing, and viscoplastic deformation. The measurements are made simply, quickly and accurately. References to the many previously published works were collated to explain each of the concepts discussed here in more detail. The use of mechanics and strain decomposition simplifies the measurement techniques to the point that an entire characterization of the properties of an asphalt mixture in tension and compression can be completed in the space of one day. A few critical conclusions and discoveries are summarized as in below:

1) The fundamental crack configurations of asphalt mixtures are illustrated to be different under tensile and compressive loading, i.e., cracks grow in a direction that is perpendicular to the external tensile load, whereas cracks grow in the same direction as the external compressive load. Nevertheless, the local stresses that cause the crack opening are still tensile under both loading conditions. Fracture initiation criteria for viscoelastic materials are expressed differently under tensile and compressive loading, but both originate from the pseudo strain energy based viscoelastic Griffith fracture theory. The different expressions of the crack initiation criteria in tension and compression are caused by the different crack configurations, e.g., different material volumes to store or release energy. The tensile crack initiation criterion leads to a simple way of measuring the endurance limit. The compressive crack initiation 
criterion results in a simple way of predicting compressive strength and flow number. One layer of complexity in compression lies in the fact that the cracks beginning to grow in compression require that the material must first deform viscoplastically and then become sufficiently strain hardened.

2) A new viscoplasticity yield function, termed the Generalized Drucker-Prager (GD-P) criterion, is presented and employed in the viscoplastic evolution law, i.e., Perzyna's formula. Unlike previous models (e.g., the extended D-P model which is restricted to internal friction angles below 22 degrees), the GD-P criterion is valid for all internal friction angles of asphalt mixtures between zero and 90 degrees. The typical friction angles for asphalt mixtures are between 35 and 50 degrees. Asphalt mixtures are strainhardening materials with temperature and strain-rate dependence, which have been accounted for in the GD-P model.

3) Asphalt mixtures are significantly anisotropic in compression including inherent anisotropy (caused by preferentially oriented aggregates) and crack-induced anisotropy. The inherent anisotropy is characterized by an anisotropic vector magnitude which is used in viscoplastic damage models to address inherent anisotropy in constitutive modelling. It also fundamentally determines the slope of the viscoplastic potential function. The crack-induced anisotropy is directly addressed by the axial and radial damage density. With compressive loading, an asphalt mixture, being anisotropic, will fracture in a brittle manner with low air voids and as a ductile material with higher air voids. This is the likely explanation for the initial appearance of traffic-related fatigue cracking as a longitudinal crack in the center of the wheel path where repeated traffic loads have reduced the air void content through permanent plastic and viscoplastic deformation.

4) The use of damage density to characterize fracture in an asphalt mixture is led by the fact that fracture in asphalt mixtures is not the growth of a single crack but the simultaneous growth of multiple cracks. It was discovered that the form of the equation relating the rate of growth of damage density to the rate of change of the pseudo Jintegral per load cycle was identical with Paris' Law for a single crack. It was further discovered that the Paris' Law fracture coefficient $A$ and exponent $n$ proved to be nearly the same as for a single crack. This happy discovery made it possible to show that the linear relation between the $-\log (A)$ and $n$, which is known to exist for a single crack, also applies to the growth of damage density. The linear relation also 
applies to the damage density fracture properties in field cores which further allows the determination of the effect of field aging and moisture effects on the fracture of asphalt mixtures placed in the field.

5) The pseudo J-integral based Paris’ Law is demonstrated to be applicable to the damage density evolutionary rule under repeated compressive loading including axial and radial damage densities. The importance and usefulness of this fact for the future of asphalt mixture design cannot be overestimated. One of the first developments is that the compressive monotonic loading of cylindrical test samples permits a direct determination of the Paris' Law coefficient and exponent. It is normally expected that the measurement of $A$ and $n$ of Paris' Law requires an extended series of repeated loading. This determination that the coefficient $A$ and exponent $n$ can be determined with monotonically increasing loads makes fatigue testing of asphalt mixtures in compression dramatically simpler.

6) Healing is anti-fracture. It occurs in the rest periods between episodes of crack growth. Healing reduces the size of cracks that have developed. Healing occurs because of the combined effect of two factors: (1) recovery of internal stresses that built up during loading and (2) the mutual attraction of the crack faces. There is a short-term healing and a long term healing, the rates of which can be predicted with empirical equations which use the compliance and bond energy properties of the undamaged mixtures. The short term healing is the most important of these characteristics of a mixture in high traffic in-service conditions and depends upon the inverse of the non-polar bond energy of the mixture. A higher short-term healing rate prolongs the life of a pavement under high traffic loading rates. As expected, the healing properties vary with the binder type and air voids as well as with temperature and aging. Increasing temperature increases and increased aging reduces the rate of healing. The range of potential healing varies widely between different types of binder, making the selection of the binder for a given project, temperature and traffic level not only essential but also simple and straightforward.

The fact that the measured properties of asphalt mixtures are mechanics-based material properties makes them immediately useful as input to existing numerical modelling systems such as ABAQUS or COMSOL. Rapid and accurate predictions with these computer systems of the rates of growth of the various types of distress that are the end result of fracture, healing and viscoplastic deformation mechanisms will make possible and even 
practical the selection of the optimum mixture, pavement structure and relevant construction specifications and reliability levels that can be achieved. The further fact that all of these material properties can be measured simply, accurately, and rapidly and can be completed in a single day makes the entire process of mixture and pavement structural design, formulation of construction specifications and determination of a realistic and achievable reliability both efficient and cost-effective.

\section{References}

1. Abdulshafi A and Majidzadeh K. (1984). Combo Viscoelastic-pastic Modeling and Rutting of Asphalt Mixtures. Transportation Research Record: Journal of the Transportation Research Board, 968(1): 19-31.

2. Abu Al-Rub RK, Darabi MK, Huang C-W, Masad EA, and Little DN. (2012). Comparing Finite Element and Constitutive Modelling Techniques for Predicting Rutting of Asphalt Pavements. International Journal of Pavement Engineering, 13(4): 322-338.

3. Abu Al-Rub RK and Voyiadjis GZ. (2003). On the Coupling of Anisotropic Damage and Plasticity Models for Ductile Materials. International Journal of Solids and Structures, 40(11): 2611-2643.

4. Chaboche JL. (1987). Continuum Damage Mechanics: Present State and Future Trends. Nuclear Engineering and Design, 105(1): 19-33.

5. Cheng D, Little D, Lytton R, and Holste JC. (2002). Surface Energy Measurement of Asphalt and Its Application to Predicting Fatigue and Healing in Asphalt Mixtures. Transportation Research Record: Journal of the Transportation Research Board, 1810(1): 44-53.

6. Gibson NH, Schwartz CW, Schapery RA and Witczak MW. (2003). Viscoelastic, Viscoplastic, and Damage Modeling of Asphalt Concrete in Unconfined Compression. Transportation Research Record: Journal of the Transportation Research Board, 1860(1): 3-15.

7. Griffith AA. (1921). The Phenomena of Rupture and Flow in Solids. Philosophical Transactions of the Royal Society A, 221: 163-198.

8. Gu F, Zhang Y, Luo X, Luo R and Lytton RL. (2014). Improved Methodology to Evaluate Fracture Properties of Warm-Mix Asphalt Using Overlay Test. 
Transportation Research Record: Journal of the Transportation Research Board, 2506(1): 8-18.

9. Gu F, Luo X, Zhang Y and Lytton RL. (2015). Using Overlay Test to Evaluate Fracture Properties of Field-Aged Asphalt Concrete. Construction and Building Materials, 101: 1059-1068.

10. Jacobs MMJ. (1995). Crack Growth in Asphaltic Mixes. Ph.D. Dissertation, the Delft University of Technology, The Netherlands.

11. Kachanov LM. (1986). Introduction to Continuum Damage Mechanics, New York, NY: Springer, LLC.

12. Kim YR, Little DN and Benson FC. (1990). Chemical and Mechanical Evaluation of Healing Mechanisms in Asphalt Concrete. Journal of Association of Asphalt Paving Technologists, 59: 240-275.

13. Kuai H, Lee HJ, Zi G and Mun S. (2009). Application of Generalized J-Integral to Crack Propagation Modeling of Asphalt Concrete Under Repeated Loading. Transportation Research Record: Journal of the Transportation Research Board, 2127(1): 72-81.

14. Lemaitre J and Desmorat R. (2005). Engineering Damage Mechanics: Ductile, Creep, Fatigue and Brittle Failures, New York, NY: Springer, LLC.

15. Luo X, Luo R and Lytton RL. (2013a). Characterization of Asphalt Mixtures Using Controlled-Strain Repeated Direct Tension Test. Journal of Materials in Civil Engineering, 25(2): 194-207.

16. Luo X, Luo R and Lytton RL. (2013b). Characterization of Fatigue Damage in Asphalt Mixtures Using Pseudo Strain Energy. Journal of Materials in Civil Engineering, 25(2): 208-218.

17. Luo X, Luo R and Lytton RL. (2013c). Characterization of Recovery Properties of Asphalt Mixtures. Construction and Building Materials, 48: 610-621.

18. Luo X, Luo R and Lytton RL. (2013d). Modified Paris' Law to Predict Entire Crack Growth in Asphalt Mixtures. Transportation Research Record: Journal of the Transportation Research Board, 2373(1): 54-62.

19. Luo X, Luo R and Lytton RL. (2013e). Energy-Based Mechanistic Approach to Characterize Crack Growth of Asphalt Mixtures. Journal of Materials in Civil Engineering, 25(9): 1198-1208. 
20. Luo X, Luo R and Lytton RL. (2014a). Energy-Based Crack Initiation Criterion for Viscoelastoplastic Materials with Distributed Cracks. Journal of Engineering Mechanics, 141(2): 04014114.

21. Luo X, Luo R and Lytton RL. (2014b). Energy-Based Mechanistic Approach for Damage Characterization of Pre-Flawed Visco-Elasto-Plastic Materials. Mechanics of Materials, 70: 18-32.

22. Luo X, Luo R and Lytton RL. (2015a). Mechanistic Modeling of Healing in Asphalt Mixtures Using Internal Stress. International Journal of Solids and Structures, 60: 3547.

23. Luo X, Zhang Y and Lytton RL. (2015b). Implementation of Pseudo J-Integral Based Paris' Law for Fatigue Cracking in Asphalt Mixtures and Pavements. Materials and Structures, In Press.

24. Luo X and Lytton RL. (2016). Characterization of Healing of Asphalt Mixtures Using Creep and Step-Loading Recovery Test. Journal of Testing and Evaluation, 44(6): 0090-3973.

25. Lytton RL, Roque RL, Uzan J, Hiltunen DR, Fernando E and Stoffels SM. (1993). Development and Validation of Performance Prediction Models and Specifications for Asphalt Binders and Paving Mixes. Report No. SHRP-A-357, Washington DC.

26. Lytton RL, Masad EA, Zollinger C, Bulut R, and Little D. (2005). Measurements of Surface Energy and Its Relationship to Moisture Damage. Report No. FHWA/TX05/0-4524-2, College Station, Texas.

27. Lytton RL, Gu F, Zhang Y, and Luo X. (2017). Characteristics of Undamaged Asphalt Mixtures in Tension and Compression. International Journal of Pavement Engineering, in press.

28. Marek, CR, and Herrin M. (1968). Tensile Behavior and Failure Characteristics of Asphalt Cements in Thin Films. Proc Assoc Asphalt Paving Technol., 37, 386-421.

29. Masad E, Dessouky S and Little D. (2007). Development of An Elastoviscoplastic Microstructural-based Continuum Model to Predict Permanent Deformation in Hot Mix Asphalt. International Journal of Geomechanics, 7(2): 119-130.

30. Oda M and Nakayama H. (1989). Yield Function for Soil with Anisotropic Fabric. Journal of Engineering Mechanics, 115(1): 89-104.

31. Paris PC and Erdogan F. (1963). A Critical Analysis of Crack Propagation Laws. Journal of Basic Engineering, 85: 528-534. 
32. Perzyna P. (1971). Thermodynamic Theory of Viscoplastcity. Advances in Applied Mechanics, 11(1): 313-354.

33. Ramberg W and Osgood WR. (1943). Description of Stress-Strain Curves by Three Parameters. NACA Technical Note No. 902, Washington DC.

34. Schapery RA. (1984). Correspondence Principles and a Generalized J Integral for Large Deformation and Fracture Analysis of Viscoelastic Media. International Journal Fracture, 25: 195-223.

35. Seibi A, Sharma M, Ali G and Kenis W. (2001). Constitutive Relations for Asphalt Concrete Under High Rates of Loading. Transportation Research Record: Journal of the Transportation Research Board. 1767(1): 111-119.

36. Tan S, Low B and Fwa T. (1994). Behavior of Asphalt Concrete Mixtures in Triaxial Compression. Journal of Testing and Evaluation, 22(3): 195-203.

37. Tashman L, Masad E, Little D and Zbib H. (2005). A Microstructure-based Viscoplastic Model for Asphalt Concrete. International Journal of Plasticity, 21(9): 1659-1685.

38. Tong Y. (2013). Fatigue Resistance of Asphalt Mixtures Affected by Water Vapor Movement. Ph.D. Dissertation, Texas A\&M University, College Station, Texas.

39. Underwood B, Yun T and Kim Y. (2011). Experimental Investigations of the Viscoelastic and Damage Behaviors of Hot-Mix Asphalt in Compression. Journal of Materials in Civil Engineering, 23(4): 459-466.

40. Voyiadjis GZ and Park T. (1997). Local and Interfacial Damage Analysis of Metal Matrix Composites Using the Finite Element Method. Engineering Fracture Mechanics, 56(4): 483-511.

41. Wang LB, Myers LA, Mohammad LN and Fu YR. (2003). Micromechanics Study on Top-Down Cracking. Transportation Research Record: Journal of the Transportation Research Board, 2976(1): 121-133.

42. Williams D, Little DN, Lytton RL, Kim YR and Kim Y. (2001). Microdamage Healing in Asphalt Concrete, Volume II: Laboratory and Field Testing to Assess and Evaluate Microdamage and Microdamage Healing. Report No. DTFH61-92-C-00170, FHWA, U.S. Department of Transportation.

43. Zhang Y, Luo R and Lytton RL. (2011). Microstructure-Based Inherent Anisotropy of Asphalt Mixtures. Journal of Materials in Civil Engineering, 23(10): 1473-1482. 
44. Zhang Y, Luo R and Lytton RL. (2012). Characterizing Permanent Deformation and Fracture of Asphalt Mixtures by Using Compressive Dynamic Modulus Tests. Journal of Materials in Civil Engineering, 24(7): 898-906.

45. Zhang Y. (2012). Anisotropic Characterization of Asphalt Mixtures in Compression. Ph.D. Dissertation, College Station, Texas, USA: Texas A\&M University.

46. Zhang Y, Luo R and Lytton RL. (2013a). Characterization of Viscoplastic Yielding of Asphalt Concrete. Construction and Building Materials, 47: 671-679.

47. Zhang Y, Luo R and Lytton RL. (2013b). Mechanistic Modeling of Fracture in Asphalt Mixtures under Compressive Loading. Journal of Materials in Civil Engineering, 25(9): 1189-1197.

48. Zhang Y, Bernhardt M, Biscontin G, Luo R and Lytton RL. (2014a). A Generalized Drucker-Prager Viscoplastic Yield Surface Model for Asphalt Concrete. Materials and Structures, 48(11): 3585-3601.

49. Zhang Y, Luo R and Lytton RL. (2014b). Anisotropic Characterization of Crack Growth in Tertiary Flow of Asphalt Mixtures in Compression. Journal of Engineering Mechanics, 140(6): 04014032.

50. Zhang Y, Luo X, Luo R and Lytton RL. (2014c). Crack Initiation in Asphalt Mixtures under External Compressive Loads. Construction and Building Materials, 72: 94-103.

51. Zhang Y, Birgisson B, Gu F and Lytton RL. (2016). Crack Evolution of Asphalt Mixtures under Compressive Monotonic and Repeated Loads. The 8th Rilem International Conference on Mechanisms of Cracking and Debonding in Pavements (MCD2016), Nantes, France.

52. Zhang Y, Gu F, Birgisson B, and Lytton RL. (2017). Viscoelasticplastic-Fracture Modeling of Asphalt Mixtures Under Monotonic and Repeated Loads. Transportation Research Record: Journal of the Transportation Research Board, 2631(1): 20-29. 